\title{
Use of Physiologically Based Pharmacokinetic (PBPK) Modeling for Predicting Drug-Food Interactions: an Industry Perspective
}

\author{
Arian Emami Riedmaier, ${ }^{1,24}$ Kevin DeMent, ${ }^{2}$ James Huckle, ${ }^{3}$ Phil Bransford, ${ }^{4}$ Cordula Stillhart, ${ }^{5}$ Richard Lloyd, ${ }^{6}$ \\ Ravindra Alluri, ${ }^{7}$ Sumit Basu, ${ }^{8}$ Yuan Chen, ${ }^{9}$ Varsha Dhamankar, ${ }^{10,11}$ Stephanie Dodd, ${ }^{12}$ Priyanka Kulkarni, ${ }^{13}$ \\ Andrés Olivares-Morales, ${ }^{14}$ Chi-Chi Peng, ${ }^{13,15}$ Xavier Pepin, ${ }^{16}$ Xiaojun Ren, ${ }^{17}$ Thuy Tran, ${ }^{18}$ \\ Christophe Tistaert, ${ }^{19}$ Tycho Heimbach, ${ }^{20}$ Filippos Kesisoglou, ${ }^{21}$ Christian Wagner, ${ }^{22}$ and Neil Parrott ${ }^{23}$
}

Received 29 June 2020; accepted 1 September 2020; published online 27 September 2020

\begin{abstract}
The effect of food on pharmacokinetic properties of drugs is a commonly observed occurrence affecting about $40 \%$ of orally administered drugs. Within the pharmaceutical industry, significant resources are invested to predict and characterize a clinically relevant food effect. Here, the predictive performance of physiologically based pharmacokinetic (PBPK) food effect models was assessed via de novo mechanistic absorption models for 30 compounds using controlled, pre-defined in vitro, and modeling methodology. Compounds for which absorption was known to be limited by intestinal transporters were excluded in this analysis. A decision tree for model verification and optimization was followed, leading to high, moderate, or low food effect prediction confidence. High (within 0.8- to 1.25-fold) to moderate confidence (within 0.5- to 2-fold) was achieved for most of the compounds (15 and 8, respectively). While for 7 compounds, prediction confidence was found to be low (>2-fold). There was no clear difference in prediction success for positive or negative food effects and no clear relationship to the BCS category of tested drug molecules. However, an association could be demonstrated when the food effect was mainly related to
\end{abstract}

Guest Editor: Filippos Kesisoglou

Electronic supplementary material The online version of this article (https://doi.org/10.1208/s12248-020-00508-2) contains supplementary material, which is available to authorized users.

${ }^{1}$ DMPK and Translational Modeling, AbbVie Inc., North Chicago, Illinois, USA.

${ }^{2}$ Global DMPK, Takeda Pharmaceutical Co., Ltd., San Diego, California, USA.

${ }^{3}$ Drug Product Technology, Amgen, Thousand Oaks, California, USA.

${ }^{4}$ Modeling \& Informatics, Vertex Pharmaceuticals, Boston, Massachusetts, USA.

${ }^{5}$ Pharmaceutical R\&D, Formulation \& Process Sciences, F. Hoffmann-La Roche Ltd., Basel, Switzerland.

${ }^{6}$ Computational \& Modelling Sciences, Platform Technology Sciences, GlaxoSmithKline R\&D, Ware, Hertfordshire, UK.

${ }^{7}$ Clinical Pharmacology and Safety Sciences, R\&D, AstraZeneca, Cambridge, UK.

${ }^{8}$ Pharmacokinetic, Pharmacodynamic and Drug MetabolismQuantitative Pharmacology and Pharmacometrics (PPDM-QP2), Merck \& Co, Inc., West Point, Pennsylvania, USA.

${ }^{9}$ Department of Drug Metabolism and Pharmacokinetics, Genentech, South San Francisco, California, USA.

${ }^{10}$ Formulation Development, Vertex Pharmaceuticals, Boston, Massachusetts, USA.

${ }^{11}$ Present Address: Formulation Development, Cyclerion Therapeutics Inc., Cambridge, Massachusetts, USA.
${ }^{12}$ Chemical \& Pharmaceutical Profiling, Novartis Institutes for Biomedical Research, Cambridge, Massachusetts, USA.

${ }^{13}$ Department of Pharmacokinetics and Drug Metabolism, Amgen Inc., Cambridge, Massachusetts, USA.

${ }^{14}$ Pharmaceutical Sciences, Roche Pharmaceutical Research and Early Development, Roche Innovation Center, Basel, Switzerland.

${ }^{15}$ Present Address: Drug Metabolism and Pharmacokinetics, Theravance Biopharma, South San Francisco, California, USA.

${ }^{16}$ New Modalities and Parenteral Development, Pharmaceutical Technology \& Development, Operations, AstraZeneca, Macclesfield, UK.

${ }^{17}$ Modeling \& Simulation, PK Sciences, Novartis Institutes of Biomedical Research, East Hanover, New Jersey, USA.

${ }^{18}$ Computational \& Modelling Sciences, Platform Technology Sciences, GlaxoSmithKline R\&D, Collegeville, Pennsylvania, USA.

${ }^{19}$ Pharmaceutical Sciences, Janssen Research \& Development, Beerse, Belgium.

${ }^{20}$ PBPK \& Biopharmaceutics, Novartis Institutes of Biomedical Research, Wayne, New Jersey, USA.

${ }^{21}$ Pharmaceutical Sciences, Merck \& Co., Inc., Kenilworth, New Jersey, USA.

${ }^{22}$ Pharmaceutical Technologies, Chemical and Pharmaceutical Development, Merck Healthcare KGaA, Darmstadt, Germany.

${ }^{23}$ Pharmaceutical Sciences, Roche Pharmaceutical Research and Early Development, Roche Innovation Center, Basel, Switzerland.

${ }^{24}$ To whom correspondence should be addressed. (e-mail: arian.emamiriedmaier@abbvie.com) 
changes in the gastrointestinal luminal fluids or physiology, including fluid volume, motility, $\mathrm{pH}$, micellar entrapment, and bile salts. Considering these findings, it is recommended that appropriately verified mechanistic PBPK modeling can be leveraged with high to moderate confidence as a key approach to predicting potential food effect, especially related to mechanisms highlighted here.

KEY WORDS: drug-food interaction ; food effect; modeling and simulation; PBBM ; PBPK.

\section{INTRODUCTION}

For orally administered drugs, the consumption of food at the time of drug administration can alter absorption (1). Food effect is widespread, and over $40 \%$ of the orally administered drugs approved by the FDA or EMA in the last 10 years were reported to have altered pharmacokinetics (PK) by food (2). Therefore, health authorities expect sponsors to characterize food or meal effects prior to approval $(3,4)$. While a food effect assessment is typically performed with a standardized high-fat meal, in certain cases, evaluation of different meal types (i.e., varying macro and calorie contents) may be recommended. If food does not have a clinically significant impact on PK, then sponsors can conduct pivotal trials without regard to food and labeling can state that the drug may be taken with or without food. However, in the case of a clinically meaningful PK effect, a specific recommendation will be made for drug administration; for example, a drug can only be given under fasted conditions or taken with a meal to maximize drug absorption.

Recently updated FDA guidance on the conduct of food effect studies describe, in detail, the clinical study design, data analysis, and labeling recommendation. Notably, neither the recent FDA guidance nor guidance from other agencies has mentioned the utility of mechanistic studies of food effect using in vitro and in silico models. However, within the pharmaceutical industry, significant resources are often invested to anticipate, characterize, and mitigate a food effect, since clinical studies alone do not provide the mechanistic insights needed to predict and understand food effects (2). Recent reviews have covered the multiple mechanisms for food effect (5) and the tools available to understand them (6). Among them, PBPK modeling has gained critical attention for the prediction of food effects using the advanced absorption models in commercial software platforms such as GastroPlus ${ }^{\mathrm{TM}}$ and Simcyp ${ }^{\circledR}$ (7-9). However, despite these examples, the health authorities still lack confidence in these predictions $(8,10)$. This may, in part, be due to a lack of best practices for consistent modeling strategy, as well as, lack of a prospective approach to evaluating the success of food effect predictions.

Therefore, this work aims to assess the predictive performance of PBPK food effect models and to provide recommendation for best practice. Instead of relying on previously published models, the Food Effect PBPK IQ Working Group generated de novo mechanistic absorption models for 30 compounds using physicochemical and in vitro data generated in accordance with pre-defined methodology. Furthermore, a decision tree is proposed for model verification and optimization, which was strictly followed within the working group. Thus, this work provides a well-controlled assessment of PBPK food effect modeling by minimizing confounding factors, such as inconsistent data generation, subjective model verification/optimization, and variable modeler experiences.

\section{MATERIALS AND METHODS}

To reduce methodological bias in PBPK input parameters, this work mostly used permeability, solubility, surface $\mathrm{pH}$, and dissolution data generated using pre-defined methods by this working group. However, if literature data used comparable methods, values were not re-measured. This information is indicated in Table I.

\section{Compound Selection}

A comprehensive compound list with clinically observed PK changes in the presence of food was collated through a detailed literature search and curation (Supplementary Table 1). The information collected included the outcome of a food effect study, the compound type (i.e., acid, base, or ampholyte), and proposed mechanism of food effect. To focus on absorption-related mechanisms of food effect and to reduce variability in modeling where there is low confidence in the disposition of a given compound, all compounds lacking clinical intravenous (IV) PK data or population PKbased data, as well as compounds with high hepatic extraction, were excluded from this list. Furthermore, prodrugs and compounds whose absorption is known to be limited by active transport were excluded, though these compounds are not expected to make up a large subset of clinical compounds displaying food effect (Supplementary Table 1). The compound list was subsequently refined to 30 compounds for final modeling and analysis while ensuring equal distribution of compound, BCS, and food effect type (Table I, Fig. 1). Food effect (FE) type was defined based on AUC and/or $C_{\max }$ ratios of fed to fasted using $\mathrm{BE}$ criteria (i.e., within 0.8-1.25). FE definitions were based on the drug label and set to positive if the ratio of fed $>$ fasted, negative if ratio fasted $>$ fed, or none if no significant change in AUC and $C_{\max }$ with food.

\section{Permeability Measurement in MDCK Cells}

Wild-type Madin-Darby canine kidney (MDCK-WT) cell line was obtained from NKI (Amsterdam, The Netherlands) and modified to knockdown endogenous canine $\mathrm{P}$ glycoprotein (P-gp). Permeability through a cell monolayer was determined with a Transwell ${ }^{\mathrm{TM}}$ system. Cells were plated on the apical side of 96-well Transwell plates 4-7 days prior to the experiment and were cultured at $37^{\circ} \mathrm{C}$ under a $5 \% \mathrm{CO}_{2}$ atmosphere. All compounds were dissolved in Hanks' balanced salt solution (HBSS) plus $80 \mathrm{mM}$ Lucifer Yellow (LuY) and $10 \mu \mathrm{M}$ cyclosporin $\mathrm{A}$, which was added to the apical 


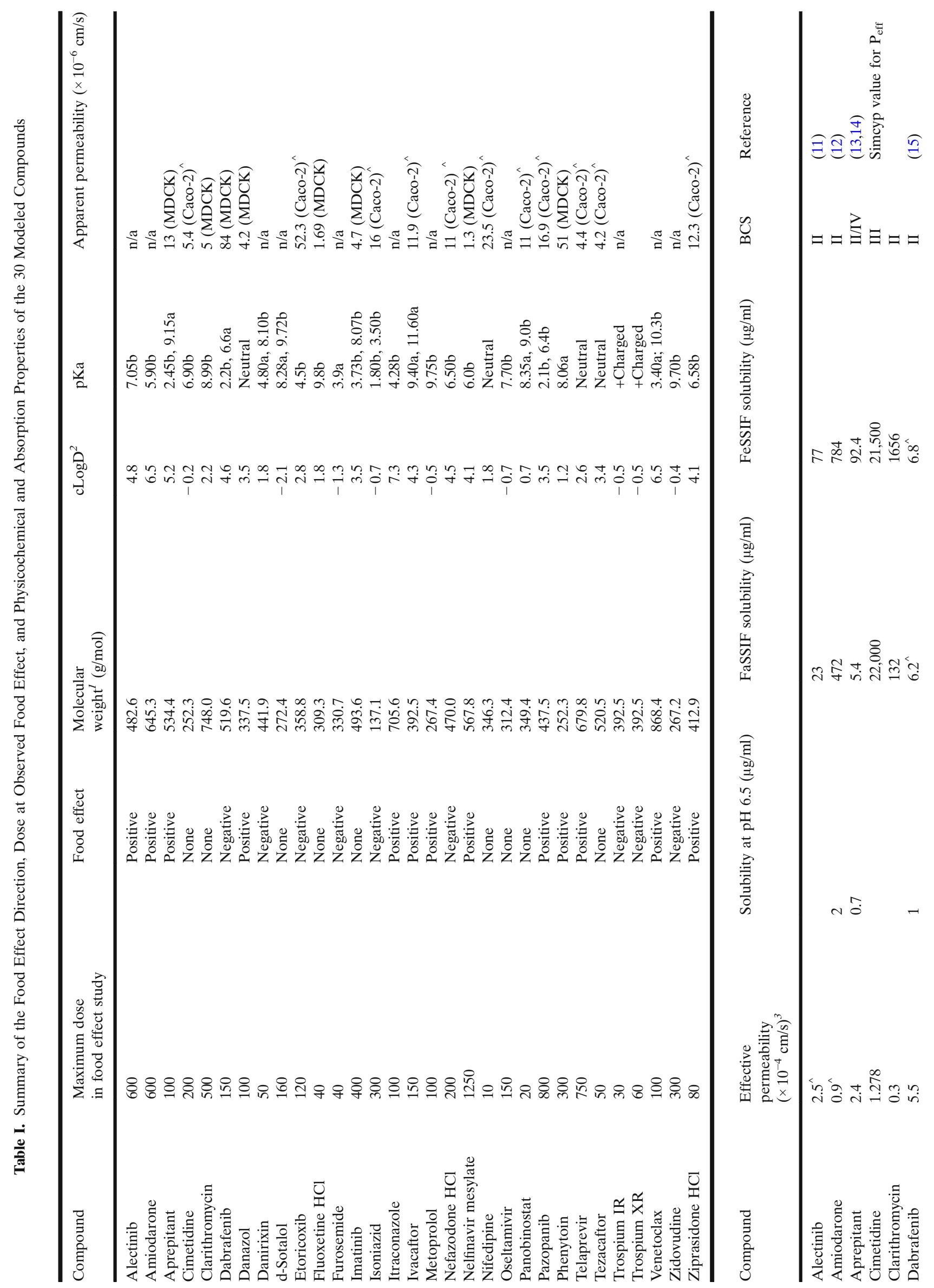




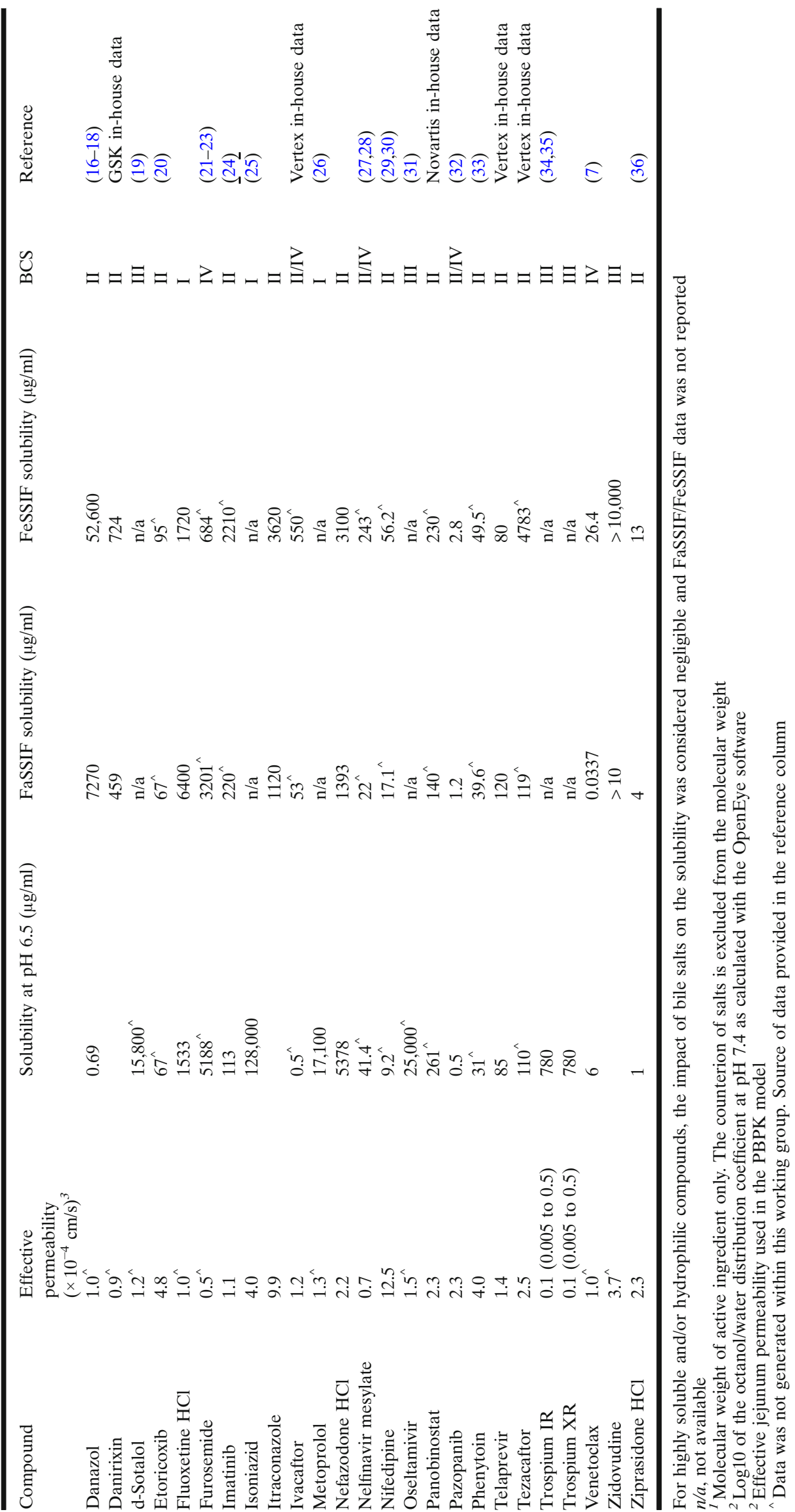




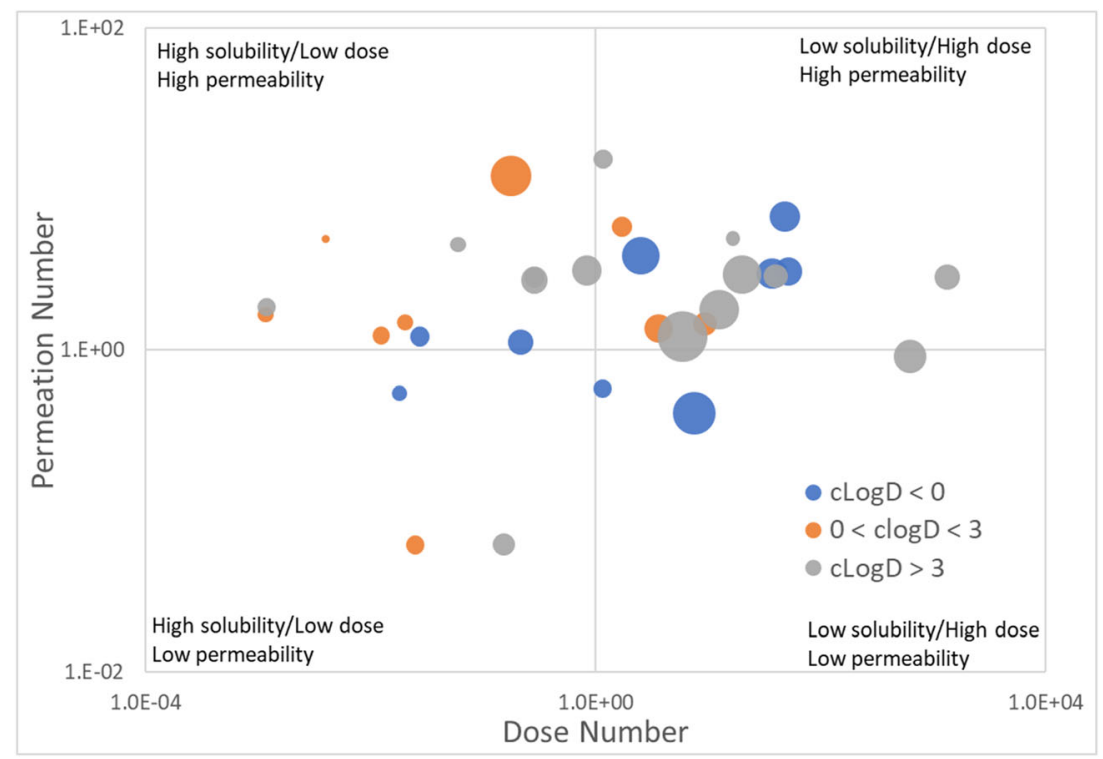

Fig. 1. Physicochemical properties of the 30 modeled compounds. The compounds selected cover a range of solubility, permeability, molecular weight, and lipophilicity. A compound's unitless dose number is calculated as the maximum dose administered in the food effect study in $\mathrm{mg}$, divided by the FaSSIF or buffer solubility in $\mathrm{mg} / \mathrm{ml}$, and divided by an approximate small intestine fluid volume of $500 \mathrm{ml}$. A dose number greater than 1 indicates low solubility or a high dose while a dose number less than one indicates high solubility or low dose. The unitless permeation number is calculated as the effect jejunum permeability multiplied by the surface-to-volume ratio of the small intestine assuming a $1.75 \mathrm{~cm}$ cylindrical radius, multiplied by the small intestine transit time assumed to be $3 \mathrm{~h}$. A permeation number greater than one indicates high permeability while a permeability less than 1 indicates poor permeability. The size of the markers is proportional to the active ingredient's molecular weight. The color encodes the calculated lipophilicity

wells. The corresponding receiver (basolateral) wells were filled with HBSS plus $10 \mu \mathrm{M}$ cyclosporin A, a P-gp Inhibitor.

Permeation rates of the compounds, including reference compounds, were measured in the apical-to-basolateral (AB) direction. The donor and receiver wells were sampled immediately after application of the compound to the donor well to determine baseline concentrations, and again after $1 \mathrm{~h}$. Quantification was done using high-performance liquid chromatography combined with mass spectrometry (HPLC-MS/ MS) analysis and monolayer integrity was verified by analyzing the receiver samples for LuY fluorescence in a plate reader. Control compounds were run in parallel to test compounds and were used to scale the apparent permeability $\left(P_{\text {app }} \times 10^{-6} \mathrm{~cm} / \mathrm{s}\right)$ to an effective human permeability $\left(P_{\text {eff,man }} \times 10^{-4} \mathrm{~cm} / \mathrm{s}\right)$ using the software's built-in calibration curve (21).

\section{Solubility Measurement in Aqueous Buffer Solutions and Biorelevant Media}

The solubility of the drug substances was determined in biorelevant media as well as in aqueous buffers at different pH. Fasted state simulated gastric fluid (FaSSGF) (37), fasted state simulated intestinal fluid (FaSSIF-V2) (38), and fed state simulated intestinal fluid (FeSSIF-V2) (39) were prepared according to the instructions provided by Biorelevant (Biorelevant.com Ltd., London, UK). Hydrochloric acid pH 2, citrate buffer $\mathrm{pH} 4$, and phosphate buffer $\mathrm{pH} 7$, as well as additional buffer solutions if required, were prepared according to the standard buffer solutions described in the United States Pharmacopeia (USP) [USP 41, buffer solutions, 5748-5749]. For neutral compounds, solubility was determined at $\mathrm{pH} \mathrm{2,} \mathrm{pH} 4$, and $\mathrm{pH} 7$. For ionizable compounds, two additional solubility data points, one $\mathrm{pH}$ unit above and below the pKa value(s), were collected.

Excess of drug substance was equilibrated in the media on a magnetic stirrer $(200 \mathrm{rpm})$ at $37^{\circ} \mathrm{C}$ (biorelevant media) and at room temperature (aqueous buffer). The concentration of dissolved drug and the medium $\mathrm{pH}$ were determined after 1, 2, 6, and $24 \mathrm{~h}$. The equilibrium solubility was interpreted as the concentration measured after a plateau was reached and was at the latest measured after $24 \mathrm{~h}$. For freely soluble compounds, the extent of solubilization was measured only up to $10 \mathrm{mg} / \mathrm{mL}$.

\section{PBPK Modeling Approach}

An aligned decision tree was defined by working group members prior to modeling, as outlined in Fig. 2. In short, PBPK models were built for all compounds in Simcyp V17.1 (Certara, USA, Inc.) and/or GastroPlus V9.5 (Simulations Plus, Inc.). A software comparison was not the aim of this working group. However, if the results of the two software platforms (i.e., model 1 vs. model 2) showed any large discrepancies, this was reported (Table II, footnotes), and where possible, the underlying mechanisms were investigated and described (Table III, Fig. 5). For GastroPlus, individual, population-representative simulations were 


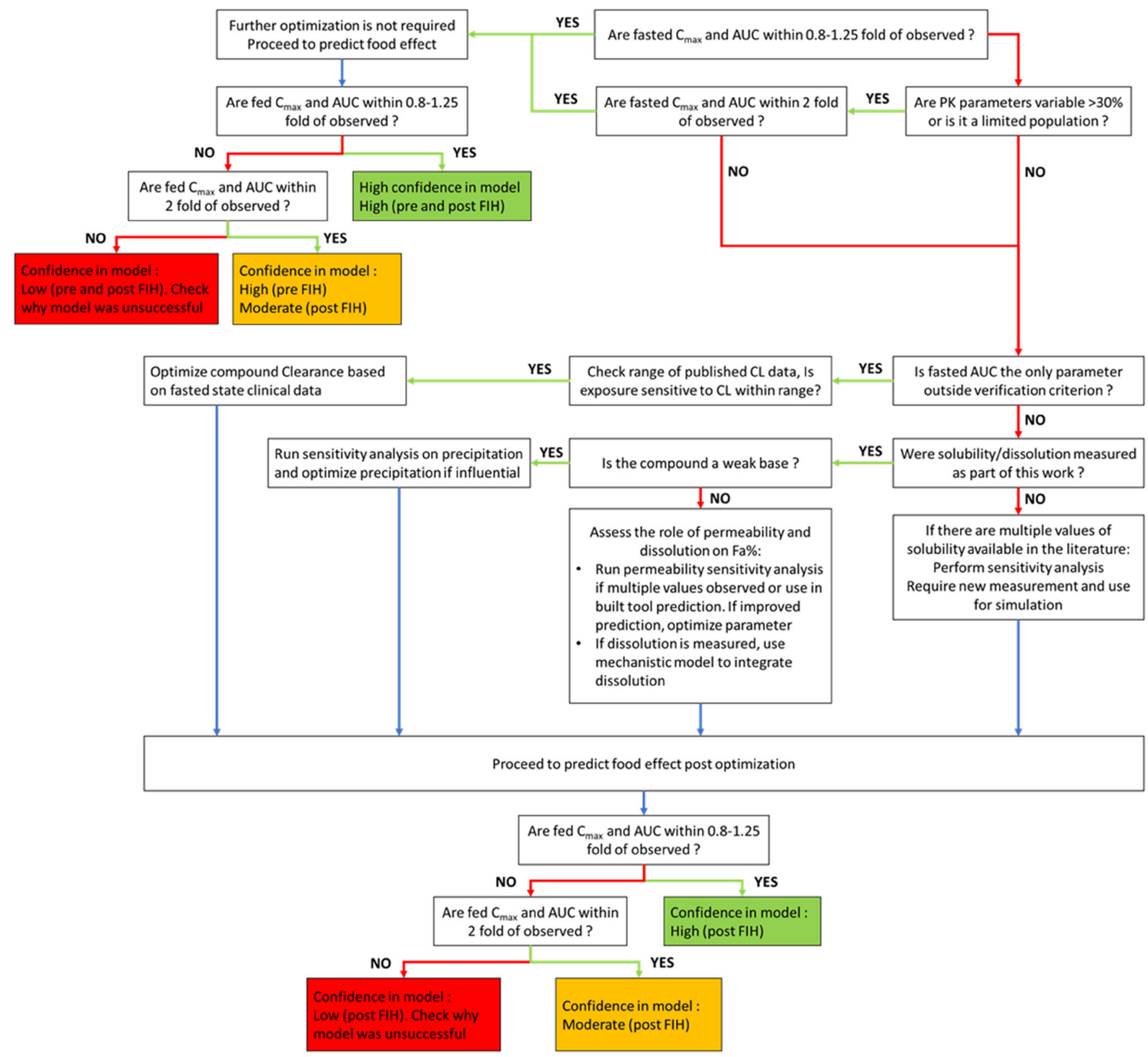

Fig. 2. Decision tree for the verification and optimization of food effect projections using PBPK. This decision tree was utilized by all modelers working on this initiative to verify and, if necessary, optimize their models using an aligned and consistent approach. Confidence categories were defined based on the outcome of this workflow after an independent review of the model outcome and verification. A summary of the outcome of PBPK modeling based on this decision tree for the 30 compounds is provided in Table II

conducted as best practice. For Simcyp, all simulations were run in the healthy volunteer population using the default system parameters and with the clinical trial design and doses matched to the reported studies. Published and measured values for physicochemical properties, permeability, solubility, and dissolution were utilized as input parameters, respectively, to build mechanistic, bottom-up models for absorption. In order to reduce the uncertainty and variability and narrow the analysis of food effect predictions to absorption-related mechanisms, clearance and disposition were modeled based on published clinical IV PK and/or population PK data. This was not done because IV data is required or recommended by the working group for PBPK model success, but rather to simplify the modeling approach and subsequent analyses, such that model outcomes could be interpreted in the context of absorption parameters only. Furthermore, the focus of this work was to study FE related to absorption mechanisms and not, for example, hepatic first pass or metabolism changes.

The decision tree outlined specific criteria for a model to be considered verified, as well as potential steps to optimize a model where necessary (Fig. 2). The decision tree was followed by all modelers and used to determine the degree of success in predicting food effect. Parameters that were optimized were limited to clearance, precipitation time, and/ or permeability. Due to uncertainty in the bio-relevance of in vitro solubility data, solubility was not optimized once the relevant solubility input was evaluated by comparing the 
different in vitro measured solubility values. In some cases where the decision tree did not lead to a successful model even after optimization, additional steps were taken to optimize the model to enable hypothesis testing; these examples are further discussed in an accompanying manuscript in this issue (e.g., discussion on pazopanib).

\section{Confidence Criteria}

PBPK models were developed based on the decision tree outlined in Fig. 2, initially using a bottom-up approach and subsequently using a middle-out approach for cases where verification based on the decision tree criteria was not successful. Compounds were assigned to a pair of modelers with one modeler building the model and the other reviewing it for accuracy and goodness of fit. Success was defined based on visual inspection of the PK profile overlay (i.e., if there was a $T_{\max }$ or $C_{\max }$ shift), as well as quantitative assessment of $C_{\max }$ and AUC ratios (verification range defined in Fig. 1 and described in more detail below).

Model performance was evaluated in the context of the stage of drug development (i.e., purely bottom-up vs. middleout) using two key criteria: confidence in predicting the likelihood of food effect (i.e., risk assessment) and confidence in predicting the direction and extent of food effect.

The first criterion was assessed using a qualitative yes/no categorization in answer to the question: was the food effect captured correctly in the absence of model optimization with clinical data?

The second criterion was quantitative in nature and involved evaluation of observed versus predicted AUC and $C_{\text {max }}$ ratios of fasted and fed.

When the bottom-up model could accurately capture the fasted and fed PK parameters and profile within 2-fold of observed, and visual inspection indicated good overlay of the PK profiles without the need for optimization of absorption parameters, the model was considered to have high confidence with respect to bottom-up success (confidence category: high confidence bottom-up). Second, where the bottomup model could accurately capture the fasted and fed PK parameters and profile within $0.8-1.25$ range, but only after optimization of absorption parameter(s) as defined in the decision tree, the model was considered high confidence with respect to middle-out success, e.g., for informing food effect of new formulations and dose strengths (confidence category: high confidence middle-out). Third, where the model could capture the fasted and fed PK parameters and profile following optimization using fasted data, though it fell outside the conservative criteria defined above, but within 2-fold of observed PK parameters, the model was considered to have moderate confidence. Finally, where the model failed to capture the fasted and/or fed PK parameters and profile even after optimization as described in the decision tree, it was categorized as low confidence (Fig. 2). While modeling the latter subset of compounds using a broad, pre-defined decision tree around optimization was not found to be suitable, deviating from the general workflow helped improve the accuracy of some of the models; these examples are captured in an accompanying manuscript in this issue focusing on low confidence predictions.

\section{RESULTS}

Based on the exclusion criteria described above, 30 compounds were selected for modeling. The selected compounds showed diverse properties and included 13 compounds with positive food effect, 8 compounds with negative food effect, and 9 compounds with no food effect. Of the 30 compounds modeled, 24 of the models could correctly capture the likelihood of food effect without any optimization, this is indicated by a yes/no on Table II (i.e., risk assessment) (Table II). Furthermore, the impact of food on PK was predicted with high, moderate, and low confidence for 15, 8, and 7 compounds, respectively (Table II, Figs. 3 and 4). There was no clear correlation between the prediction confidence and BCS category and/or food effect type. However, an association could be demonstrated with the key mechanism(s) driving the food effect (Fig. 5, Table III). High confidence in PBPK prediction of food effects was typically observed for compounds where the mechanism of food effect was related to physiology, including changes in the gastrointestinal (GI) luminal fluids, fluid volume, motility, $\mathrm{pH}$, ion pairing, and bile salts. Low confidence in prediction was associated with food effects related to drug formulation interactions with the intestinal microenvironment, specifically with respect to salts and weak bases such that the model and/ or the biorelevant media used could not capture the dynamic effect of the drug on its microenvironment. Low confidence in modeling was also observed with food effects related to fedstate hydrodynamics (e.g., GI fluid viscosity) and food-drug/ micelle-drug interactions where standard in vitro assays are not able to characterize the food effect mechanistically.

Three examples from the different confidence categories have been highlighted here. For category I, a high confidence example for bottom-up application demonstrates successful verification using a purely bottom-up approach for modeling absorption and related FE. Category II exemplifies the successful verification following a middle-out approach using the optimization decision tree, identified as high confidence for post-FIH applications. Finally, an example from category III shows a failed verification following optimization using the outlined strategy. Here, confidence in using a general workflow for the prediction of food effect is low and increased confidence will depend on a case-by-case evaluation of more predictive in vitro and modeling methodologies.

\section{PBPK Predictions to Waive Food Effect Studies-Bottom- Up Application}

\section{Case Example: Nifedipine (High Confidence)}

Nifedipine is a poorly soluble, non-ionizable compound with no food effect reported on the extent of absorption when administered as a $10 \mathrm{mg}$ IR soft gelatin capsule, although the rate of absorption decreased with food (40).

The capsule contains nifedipine dissolved in an organic solvent (polyethylene glycol 400 and peppermint oil) and thus behaves like a solution.

The mechanistic, bottom-up absorption model was built using previously published solubility data (29). Precipitation time was kept at the default. The apparent Caco- 2 permeability was taken from the literature (Gertz, Harrison et al. 
Table II. Summary of the Outcome of Food Effect PBPK Modeling for 30 Compounds and the Associated Confidence in the PBPK Food Effect (FE) Prediction and Risk Assessment. The Color Coding Represents the Food Effect Direction with Green and Red Signifying Positive and Negative Food Effect, Respectively

\begin{tabular}{|c|c|c|c|c|c|c|c|c|c|c|c|c|c|c|c|c|}
\hline \multirow[t]{2}{*}{ Compound } & \multirow{2}{*}{$\begin{array}{l}\text { Food } \\
\text { Effect }\end{array}$} & \multirow[t]{2}{*}{ Model } & \multicolumn{2}{|c|}{$\begin{array}{c}\text { Simulated AUC }(0-\text { inf }) \\
\left(h^{*} n g / m L\right)\end{array}$} & 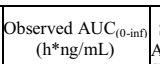 & \multirow{2}{*}{\begin{tabular}{|c|} 
Simulated \\
AUC Ratios \\
(Fed/Fasted)
\end{tabular}} & \multirow{2}{*}{$\begin{array}{l}\text { Observed } \\
\text { AUC Ratios } \\
\text { Fed/Fasted) }\end{array}$} & \multirow{2}{*}{$\begin{array}{c}\text { AUC Ratio } \\
\text { of Ratios } \\
\text { (Sim'd / Obs'd) }\end{array}$} & \multirow{2}{*}{\multicolumn{2}{|c|}{$\begin{array}{c}\text { Simulated } \\
\text { Cmax } \\
(\mathrm{ng} / \mathrm{mL})\end{array}$}} & \multirow[t]{2}{*}{$\begin{array}{c}\text { Observed } \\
\text { Cmax } \\
(\mathrm{ng} / \mathrm{mL})\end{array}$} & \multirow{2}{*}{$\begin{array}{c}\text { Simulated } \\
\text { Cmax Ratios } \\
\text { (Fed/Fasted) }\end{array}$} & \multirow{2}{*}{$\begin{array}{c}\text { Observed } \\
\text { Cmax Ratios } \\
\text { (Fed/Fasted) }\end{array}$} & \multirow{2}{*}{$\begin{array}{c}\text { Cmax Ratio } \\
\text { of Ratios } \\
\text { (Sim'd / Obs'd) }\end{array}$} & \multirow{2}{*}{$\begin{array}{c}\text { Bottom-Up } \\
\text { Risk Assessment }\end{array}$} & Confidence \\
\hline & & & Fed & Fasted & Fasted & & & & & & & & & & & \\
\hline Nelfinavir Mesylate & +++ & $\mid \begin{array}{l}\text { Model } 1 \\
\text { Model } 2\end{array}$ & $2.79 \mathrm{E}+04 \quad 9$ & $9.52 \mathrm{E}+03$ & $3.58 \mathrm{E}+047.08 \mathrm{E}+03$ & 2.93 & 5.06 & 0.58 & $2.26 \mathrm{E}+03$ & $7.79 \mathrm{E}+02 \mathrm{~B}$ & $3.91 \mathrm{E}+038.95 \mathrm{E}+02$ & 2.90 & 4.37 & 0.66 & Yes & Moderate \\
\hline & & Model 1 & $9.42 \mathrm{E}+03 \quad 5$ & $5.99 \mathrm{E}+03$ & $1.33 \mathrm{E}+043.58 \mathrm{E}+03$ & 1.57 & 3.71 & 0.42 & $7.72 \mathrm{E}+02 \quad 4$ & $4.90 \mathrm{E}+02$ & $2.08 \mathrm{E}+033.49 \mathrm{E}+02$ & 1.58 & 5.96 & 0.26 & No & Low \\
\hline Telaprevir & + & Model 2 & $8.84 \mathrm{E}+03 \quad 3$ & $3.62 \mathrm{E}+03$ & $1.33 \mathrm{E}+043.58 \mathrm{E}+03$ & 2.44 & 3.71 & 0.66 & $6.94 \mathrm{E}+02 \quad 2$ & $2.63 \mathrm{E}+022$ & $2.08 \mathrm{E}+033.49 \mathrm{E}+02$ & 2.64 & 5.96 & 0.44 & Yes & Low \\
\hline Ivacaftor & +++ & \begin{tabular}{|l|} 
Model 1 \\
Model 2
\end{tabular} & $8.90 \mathrm{E}+03 \quad 2$ & $2.81 \mathrm{E}+03$ & $9.59 \mathrm{E}+033.12 \mathrm{E}+03$ & 3.17 & 3.07 & 1.03 & $6.21 \mathrm{E}+02 \quad 1$ & $1.26 \mathrm{E}+026$ & $6.37 \mathrm{E}+021.48 \mathrm{E}+02$ & 4.93 & 4.30 & 1.15 & Yes & High (post) \\
\hline Amiodarone & +++ & \begin{tabular}{|l|} 
Model \\
Model 2
\end{tabular} & $\begin{array}{l}\text { Fasted model } 1 \\
\text { not verified } 6\end{array}$ & \begin{tabular}{|l|l|}
$1.45 \mathrm{E}+03$ \\
$6.82 \mathrm{E}+03$ \\
\end{tabular} & \begin{tabular}{|}
$3.30 E+041.40 E+04$ \\
$3.30 E+041.40 E+04$
\end{tabular} & & $\begin{array}{l}2.36 \\
2.36\end{array}$ & & $\begin{array}{l}\text { Fasted model } 1 \\
\text { not verified } 3\end{array}$ & $\begin{array}{l}11.49 \mathrm{E}+02 \\
3.82 \mathrm{E}+02\end{array}$ & $\begin{array}{l}1.40 \mathrm{E}+033.80 \mathrm{E}+02 \\
1.40 \mathrm{E}+033.80 \mathrm{E}+02\end{array}$ & & $\begin{array}{l}3.68 \\
3.68\end{array}$ & & $\begin{array}{l}\text { No } \\
\text { No }\end{array}$ & $\begin{array}{l}\text { Low } \\
\text { Low }\end{array}$ \\
\hline Venetoclax & +++ & \begin{tabular}{|l|} 
Model 1 \\
Model 2
\end{tabular} & $6.10 \mathrm{E}+03 \quad 2$ & $2.25 \mathrm{E}+03$ & $7.10 \mathrm{E}+032.08 \mathrm{E}+03$ & 2.71 & 3.41 & 0.79 & $4.00 \mathrm{E}+02 \quad 1$ & $1.60 \mathrm{E}+025$ & $5.00 \mathrm{E}+021.50 \mathrm{E}+02$ & 2.50 & 3.33 & 0.75 & Yes & Moderate \\
\hline & & Model 1 & $1.04 \mathrm{E}+04 \quad 1$ & $1.40 \mathrm{E}+03$ & $5.23 \mathrm{E}+031.79 \mathrm{E}+03$ & 7.43 & 2.92 & 2.54 & $4.10 \mathrm{E}+02 \quad 5$ & $5.00 \mathrm{E}+01$ & $2.57 \mathrm{E}+029.51 \mathrm{E}+01$ & 8.20 & 2.70 & 3.03 & Yes & Low \\
\hline Alectinib $^{1}$ & ++ & Model 2 & $3.65 \mathrm{E}+03 \quad 9$ & $9.13 \mathrm{E}+02$ & $5.23 \mathrm{E}+031.79 \mathrm{E}+03$ & 4.00 & 2.92 & 1.37 & $2.44 \mathrm{E}+02 \quad 9$ & $9.49 \mathrm{E}+012$ & $2.57 \mathrm{E}+029.51 \mathrm{E}+01$ & 2.57 & 2.70 & 0.95 & Yes & Moderate \\
\hline Danazol & ++ & \begin{tabular}{|l|} 
Model 1 \\
Model 2
\end{tabular} & \begin{tabular}{|l|} 
Fasted model \\
not verified
\end{tabular} & & $6.68 \mathrm{E}+022.31 \mathrm{E}+02$ & & 2.90 & & \begin{tabular}{|l} 
Fasted model \\
not verified
\end{tabular} & & $1.01 \mathrm{E}+023.70 \mathrm{E}+01$ & & 2.73 & & Yes & Low \\
\hline Aprepitant & ++ & \begin{tabular}{|l|} 
Model 1 \\
Model 2
\end{tabular} & $2.07 \mathrm{E}+04 \quad 8$ & $8.88 \mathrm{E}+03$ & $2.41 \mathrm{E}+048.57 \mathrm{E}+03$ & 2.33 & 2.81 & 0.83 & $8.47 \mathrm{E}+02 \quad 3$ & $3.65 \mathrm{E}+02$ & $1.10 \mathrm{E}+034.96 \mathrm{E}+02$ & 2.32 & 2.21 & 1.05 & Yes & High (post) \\
\hline & +1 & Model 1 & $3.14 E+05$ & $9.71 E+05$ & $2.02 E+068.24 E+05$ & 0.32 & 2.45 & 0.13 & $8.40 \mathrm{E}+03 \quad 3$ & $3.27 \mathrm{E}+04$ & $4.52 \mathrm{E}+042.07 \mathrm{E}+04$ & 0.26 & 2.19 & 0.12 & No & Low \\
\hline Pazopanib & & Model 2 & $5.22 E+05 \quad 5$ & $5.38 E+05$ & $\mid 2.02 E+068.24 E+05$ & 0.97 & 2.45 & 0.40 & $1.33 \mathrm{E}+04 \quad 1$ & $1.38 \mathrm{E}+04$ & $4.52 \mathrm{E}+042.07 \mathrm{E}+04$ & 0.96 & 2.19 & 0.44 & No & Low \\
\hline $\begin{array}{c}\text { Ziprasidone } \\
\mathrm{HCl}^{2}\end{array}$ & ++ & Model 1 & $502=5$ & & & & & & & & & & & & & \\
\hline $\mathrm{HCl}^{2}$ & & Model 2 & $1.23 \mathrm{E}+03 \quad 5$ & $5.80 \mathrm{E}+02$ & $1.91 \mathrm{E}+039.50 \mathrm{E}+02$ & 2.12 & 2.01 & 1.05 & $1.90 \mathrm{E}+02 \quad 1$ & $1.10 \mathrm{E}+021$ & $1.65 \mathrm{E}+028.40 \mathrm{E}+01$ & 1.73 & 1.96 & 0.88 & Yes & Moderate \\
\hline Itraconazole & +1 & Model 1 & $2.90 E+032$ & $2.14 E+03$ & $3.12 E+031.88 E+03$ & 1.35 & 1.66 & 0.82 & $2.16 \mathrm{E}+02 \quad 1$ & $1.23 \mathrm{E}+02$ & $2.39 \mathrm{E}+021.40 \mathrm{E}+02$ & 1.76 & 1.71 & 1.03 & Yes & High (post) \\
\hline Itraconazole & ++ & Model 2 & $4.60 E+03 \quad 2$ & $2.77 E+03$ & $3.12 E+031.88 E+03$ & 1.66 & 1.66 & 1.00 & $2.18 \mathrm{E}+02 \quad 1$ & $1.25 \mathrm{E}+022$ & $2.39 \mathrm{E}+021.40 \mathrm{E}+02$ & 1.74 & 1.71 & 1.02 & Yes & High (post) \\
\hline & & Model 1 & $1.21 E+04 \quad 1$ & $1.32 E+04$ & $1.57 E+041.26 E+04$ & 0.92 & 1.25 & 0.74 & $1.87 \mathrm{E}+03 \quad 2$ & $2.16 \mathrm{E}+03 \mathrm{C}$ & $2.51 \mathrm{E}+031.65 \mathrm{E}+03$ & 0.87 & 1.52 & 0.57 & Yes & High (pre) \\
\hline Clarithromycin ${ }^{3}$ & ++ & Model 2 & $1.34 E+04 \quad 1$ & $1.12 E+04$ & $1.57 E+041.26 E+04$ & 1.19 & 1.24 & 0.96 & $1.79 \mathrm{E}+03 \quad 1$ & $1.57 \mathrm{E}+032$ & $2.51 \mathrm{E}+031.65 \mathrm{E}+03$ & 1.14 & 1.52 & 0.75 & Yes & Moderate \\
\hline Metoprolol & + & \begin{tabular}{|l|} 
Model 1 \\
Model 2
\end{tabular} & $2.99 E+023$ & $3.15 E+02$ & $4.25 E+023.07 E+02$ & 0.95 & 1.41 & 0.67 & $7.00 \mathrm{E}+01 \quad 9$ & $9.10 \mathrm{E}+01$ & $1.18 \mathrm{E}+028.96 \mathrm{E}+01$ & 0.77 & 1.33 & 0.58 & No & Moderate \\
\hline & + & Model 1 & $1.36 E+05 \quad 1$ & $1.17 E+05$ & $1.27 E+051.04 E+05$ & 1.17 & 1.2 & 0.95 & $6.20 \mathrm{E}+03 \quad 4$ & $4.75 \mathrm{E}+036$ & $6.95 \mathrm{E}+034.92 \mathrm{E}+03$ & 1.3 & 1.41 & 0.9 & Yes & High (post) \\
\hline Phenytoin & + & Model 2 & $1.24 E+05 \quad 1$ & $1.02 E+05$ & $1.27 E+051.04 E+05$ & 1.21 & 1.22 & 0.99 & $6.53 \mathrm{E}+03 \quad 4$ & $4.64 \mathrm{E}+036$ & $6.95 \mathrm{E}+034.92 \mathrm{E}+03$ & 1.41 & 1.41 & 1.00 & Yes & High (post) \\
\hline Tezacaftor & $+/-$ & \begin{tabular}{|l|} 
Model 1 \\
Model 2
\end{tabular} & $3.65 \mathrm{E}+03 \quad 3$ & $3.62 \mathrm{E}+03$ & $4.36 \mathrm{E}+033.97 \mathrm{E}+03$ & 1.01 & 1.10 & 0.92 & $1.77 \mathrm{E}+03 \quad 1$ & $1.78 \mathrm{E}+03$ & $1.89 \mathrm{E}+032.34 \mathrm{E}+03$ & 0.99 & 0.81 & 1.23 & Yes & High (post) \\
\hline Oseltamivir & $+/-$ & \begin{tabular}{|l|} 
Model 1 \\
Model 2
\end{tabular} & $8.70 \mathrm{E}+03 \quad 8$ & $8.20 \mathrm{E}+03$ & $6.07 \mathrm{E}+036.22 \mathrm{E}+03$ & 1.06 & 0.98 & 1.09 & $6.50 \mathrm{E}+02 \quad 6$ & $6.05 \mathrm{E}+02$ & $4.41 \mathrm{E}+025.51 \mathrm{E}+02$ & 1.07 & 0.80 & 1.34 & Yes & Moderate \\
\hline Inotinil & +1 & Model 1 & $2.21 \mathrm{E}+04 \quad 2$ & $2.21 \mathrm{E}+04$ & $43.63 \mathrm{E}+04$ & 1. & 0.91 & 1.09 & $1.33 \mathrm{E}+03 \quad 1$ & $1.68 \mathrm{E}+03$ & $032.82 \mathrm{E}+03$ & 0. & 0.8 & 0.9 & Yes & High (post) \\
\hline Imatinib & $+/-$ & Model 2 & $2.11 \mathrm{E}+04 \quad 2$ & $2.06 \mathrm{E}+04$ & $3.32 \mathrm{E}+043.63 \mathrm{E}+04$ & 1.02 & 0.91 & 1.12 & $1.66 \mathrm{E}+03 \quad 1$ & $1.76 \mathrm{E}+032$ & $2.41 \mathrm{E}+032.82 \mathrm{E}+03$ & 0.94 & 0.85 & 1.10 & Yes & High (post) \\
\hline Cimetidine & $+t$ & Model 1 & $5.06 \mathrm{E}+03 \quad 5$ & $5.22 \mathrm{E}+03$ & $4.15 \mathrm{E}+034.88 \mathrm{E}+03$ & 0.97 & 0.85 & 1.14 & $1.06 \mathrm{E}+03 \quad 1$ & $1.35 \mathrm{E}+038$ & $8.00 \mathrm{E}+028.90 \mathrm{E}+02$ & 0.79 & 0.90 & 0.87 & & High (post) \\
\hline Cimetidine & $+/-$ & Model 2 & $5.18 \mathrm{E}+03 \quad 5$ & $5.07 \mathrm{E}+03$ & $4.58 \mathrm{E}+035.35 \mathrm{E}+03$ & 1.02 & 0.86 & 1.19 & $8.19 \mathrm{E}+02 \quad 9$ & $9.10 \mathrm{E}+028$ & $8.00 \mathrm{E}+028.90 \mathrm{E}+02$ & 0.90 & 0.90 & 1.00 & Yes & High (post) \\
\hline d-Sotalol & $+/-$ & \begin{tabular}{|l|} 
Model 1 \\
Model 2
\end{tabular} & $1.22 E+04 \quad 1$ & $1.40 E+04$ & $1.26 E+041.55 E+04$ & 0.87 & 0.81 & 1.07 & $9.20 \mathrm{E}+02 \quad 1$ & $1.10 \mathrm{E}+03 \mathrm{1}$ & $1.00 \mathrm{E}+031.20 \mathrm{E}+03$ & 0.84 & 0.83 & 1.00 & Yes & High (post) \\
\hline Nefazodone IR Soln. & & \begin{tabular}{|l|} 
Model 1 \\
Model 2
\end{tabular} & & \begin{tabular}{l|}
$1.97 \mathrm{E}+03$ \\
$3.23 \mathrm{E}+03$
\end{tabular} & \begin{tabular}{l|}
$2.06 \mathrm{E}+03$ \\
$2.06 \mathrm{E}+03$
\end{tabular} & & & & & $\begin{array}{l}9.58 \mathrm{E}+02 \\
9.03 \mathrm{E}+02\end{array}$ & $\begin{array}{l}1.00 \mathrm{E}+03 \\
1.00 \mathrm{E}+03\end{array}$ & & & & & \\
\hline Nefazodone & +1 & Model 1 & $7.69 \mathrm{E}+02 \quad 9$ & $9.81 \mathrm{E}+02$ & $1.24 \mathrm{E}+031.54 \mathrm{E}+03$ & 0.78 & 0.80 & 0.98 & $2.66 \mathrm{E}+02 \quad 3$ & $3.23 \mathrm{E}+02$ & $2.95 \mathrm{E}+023.01 \mathrm{E}+02$ & 0.8 & 0. & 0.8 & Yes & High (pre) \\
\hline $\mathrm{HCl}^{4}$ & $+/-$ & Model 2 & $3.80 \mathrm{E}+03 \quad 3$ & $3.23 \mathrm{E}+03$ & $1.24 \mathrm{E}+031.54 \mathrm{E}+03$ & 1.18 & 0. & 1. & $9.28 \mathrm{E}+02$ & $9.03 \mathrm{E}+022$ & $2.95 \mathrm{E}+023.01 \mathrm{E}+02$ & 1.0 & 0 & 1.0 & Yes & Moderate \\
\hline & & Model 1 & $3.58 \mathrm{E}+04 \quad 3$ & $3.58 \mathrm{E}+04$ & $4.22 \mathrm{E}+044.44 \mathrm{E}+04$ & 1.00 & 0. & 1.05 & $1.42 \mathrm{E}+03 \quad 1$ & $1.77 \mathrm{E}+03$ & $1.44 \mathrm{E}+031.89 \mathrm{E}+03$ & 0.8 & 0. & 1.05 & Yes & High (pre) \\
\hline Etoricoxib & & Model 2 & $2.96 \mathrm{E}+04 \quad 2$ & $2.94 \mathrm{E}+04$ & $4.22 \mathrm{E}+044.44 \mathrm{E}+04$ & 10 & & 106 & $2.62 \mathrm{E}+03 \quad 3$ & $3.25 \mathrm{E}+03$ & $1.44 \mathrm{E}+031.89 \mathrm{E}+03$ & 0.8 & & 1.05 & Yes & High (pre) \\
\hline Nifedinine & - & Model 1 & $2.24 \mathrm{E}+02 \quad 2$ & $2.01 \mathrm{E}+02$ & $21.49 \mathrm{E}+021.45 \mathrm{E}+02$ & 1.11 & 1.02 & 1.09 & $6.24 \mathrm{E}+01 \quad 1$ & $1.12 \mathrm{E}+02$ & $5.87 \mathrm{E}+017.89 \mathrm{E}+01$ & 0.56 & 0.74 & 0.75 & Yes & High (pre) \\
\hline Nifedipine & & Model 2 & $1.67 \mathrm{E}+02 \quad 1$ & $1.58 \mathrm{E}+02$ & $1.49 \mathrm{E}+021.45 \mathrm{E}+02$ & 1.06 & & & $\mathrm{E}+01 \quad 7$ & & $.89 \mathrm{E}+01$ & 0.7 & 0.74 & 0.9 & Yes & High (pre) \\
\hline Fluoxetine & & Model 1 & $2.50 \mathrm{E}+03 \quad 2$ & $2.50 \mathrm{E}+03$ & $31.93 \mathrm{E}+03$ & 1. & 0. & 1. & $3.06 \mathrm{E}+01 \quad 3$ & $3.05 \mathrm{E}+013$ & $13.93 \mathrm{E}+01$ & 1. & 0 . & 1. & Yes & High (pre) \\
\hline $\mathrm{HCl}$ & - & Model 2 & $1.73 \mathrm{E}+03 \quad 2$ & $2.04 \mathrm{E}+03$ & $31.93 \mathrm{E}+03$ & 0.8 & 0. & 1. & $3.89 \mathrm{E}+01 \quad 3$ & $3.60 \mathrm{E}+013$ & $3.93 \mathrm{E}+01$ & 1.8 & 0. & 1.19 & Yes & High (pre) \\
\hline Furocemide & & Model 1 & $1.70 \mathrm{E}+03 \quad 1$ & $1.75 \mathrm{E}+03$ & $1.67 \mathrm{E}+032.09 \mathrm{E}+03$ & 0.97 & 0. & 1.21 & $3.40 \mathrm{E}+02 \quad 6$ & $6.92 \mathrm{E}+02$ & $4.28 \mathrm{E}+027.54 \mathrm{E}+02$ & 0.49 & 0. & 0.87 & Yes & High (post) \\
\hline Furosemide & & Model 2 & $3.20 \mathrm{E}+03 \quad 2$ & $2.98 \mathrm{E}+03$ & $\mid 1.59 \mathrm{E}+031.94 \mathrm{E}+03$ & 1.07 & 0. & 1. & $6.00 \mathrm{E}+02 \quad 5$ & $5.82 \mathrm{E}+024$ & $4.28 \mathrm{E}+027.54 \mathrm{E}+02$ & 1.0 & 0. & 1.82 & Yes & High (pre) \\
\hline & & Model 1 & $1.87 \mathrm{E}+02 \quad 1$ & $1.79 \mathrm{E}+02$ & $1.08 \mathrm{E}+021.28 \mathrm{E}+02$ & 1.0 & 0. & 1.2 & $7.01 \mathrm{E}+00 \quad 2$ & $2.06 \mathrm{E}+019$ & $9.80 \mathrm{E}+001.75 \mathrm{E}+01$ & 0.3 & 0. & 0.61 & Yes & High (pre) \\
\hline Panol & - & Model 2 & $1.61 \mathrm{E}+02 \quad 1$ & $1.52 \mathrm{E}+02$ & $1.08 \mathrm{E}+021.28 \mathrm{E}+02$ & 1.06 & 0.85 & 1.25 & $4.01 \mathrm{E}+01 \quad 5$ & $5.05 \mathrm{E}+019$ & $9.80 \mathrm{E}+001.75 \mathrm{E}+01$ & 0.79 & 0.56 & 1.4 & Yes & High (pre) \\
\hline Zidovudine & - & \begin{tabular}{|l|} 
Model 1 \\
Model 2
\end{tabular} & $1.58 \mathrm{E}+03 \quad 1$ & $1.58 \mathrm{E}+03$ & $2.17 \mathrm{E}+032.48 \mathrm{E}+03$ & 1.00 & 0.87 & 1.15 & $5.59 \mathrm{E}+02 \quad 1$ & $1.03 \mathrm{E}+03$ & $6.32 \mathrm{E}+021.13 \mathrm{E}+03$ & 0.54 & 0.56 & 0.97 & Yes & High (pre) \\
\hline & 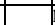 & Model 1 & $1.96 \mathrm{E}+04 \quad 2$ & $2.29 \mathrm{E}+04$ & $1.95 \mathrm{E}+042.46 \mathrm{E}+04$ & & & & $8+03 \quad 3$ & $3.95 \mathrm{E}+03 \mathrm{E}$ & $2.50 \mathrm{E}+035.00 \mathrm{E}+03$ & & & 1.4 & Yes & Moderate \\
\hline Isoniazid & - & Model 2 & $1.50 \mathrm{E}+04 \quad 2$ & $2.05 \mathrm{E}+04$ & $1.48 \mathrm{E}+041.85 \mathrm{E}+04$ & 0.73 & .00 & 91 & $2.52 \mathrm{E}+03 \quad 3$ & $3.72 \mathrm{E}+032$ & $2.73 \mathrm{E}+035.87 \mathrm{E}+03$ & 0.68 & 0.47 & 1.46 & Yes & Moderate \\
\hline Dabrofenit & ti & Model 1 & Fasted model & & & & & & Fasted model & & & & & & No & Low \\
\hline Dabrafenib & -- & Model 2 & not verified 1 & $1.81 \mathrm{E}+03$ & $8.47 \mathrm{E}+031.21 \mathrm{E}+04$ & & 0. & & not verified 2 & +021 & $1.07 \mathrm{E}+032.16 \mathrm{E}+03$ & & 0.49 & & No & Low \\
\hline Danirixin & -- & \begin{tabular}{|l|} 
Model 1 \\
Model 2
\end{tabular} & $1.47 \mathrm{E}+04 \quad 1$ & $1.50 \mathrm{E}+04$ & $1.27 \mathrm{E}+041.94 \mathrm{E}+04$ & 0.98 & 0.65 & 1.51 & $1.70 \mathrm{E}+03 \quad 2$ & $2.33 \mathrm{E}+03$ & $1.38 \mathrm{E}+033.24 \mathrm{E}+03$ & 0.73 & 0.43 & 1.70 & Yes & High (pre) \\
\hline Trosnium $\mathrm{XR}^{5}$ & & Model 1 & $8.00 \mathrm{E}+00 \quad 1$ & $1.40 \mathrm{E}+01$ & $1.42 \mathrm{E}+012.21 \mathrm{E}+01$ & & & & $3.58 \mathrm{E}-01 \quad 1$ & $1.12 \mathrm{E}+00$ & $5.42 \mathrm{E}$ & $0.3-3$ & 0.3 & 0.8 & Yes & High (post) \\
\hline Trospium XR ${ }^{3}$ & --- & Model 2 & $3.19 \mathrm{E}+00 \quad 7$ & $7.03 \mathrm{E}+01$ & $1.42 \mathrm{E}+012.21 \mathrm{E}+01$ & 0.05 & 0.64 & 0.07 & $\begin{array}{lll}5.55 \mathrm{E}-01 \quad 5 & 5\end{array}$ & $5.75 \mathrm{E}+00$ & $5.42 \mathrm{E}-011.45 \mathrm{E}+00$ & 0.10 & 0.37 & 0.26 & No & Low \\
\hline Trospium IR ${ }^{5}$ & $\ldots$ & Model 1 & $5.52 \mathrm{E}+00 \quad 2$ & $2.10 \mathrm{E}+01$ & $8.40 \mathrm{E}+005.07 \mathrm{E}+01$ & 0.26 & 0.17 & 1.59 & $2.91 \mathrm{E}-01 \quad 2$ & $2.02 \mathrm{E}+00$ & $4.68 \mathrm{E}-014.03 \mathrm{E}+00$ & 0.14 & 0.12 & 1.24 & Yes & Moderate \\
\hline Trospium IR & -- & Model 2 & $2.32 \mathrm{E}+00 \quad 4$ & $4.92 \mathrm{E}+01]_{8}$ & $|8.40 \mathrm{E}+005.07 \mathrm{E}+01|$ & 0.05 & 0.17 & 0.28 & 3.83E-01 & $3.76 \mathrm{E}+00$ & $4.68 \mathrm{E}-014.03 \mathrm{E}+00$ & 0.10 & 0.12 & 0.88 & No & Low \\
\hline
\end{tabular}

Bold italicized text indicates $\operatorname{AUC}(0-\mathrm{t})$, not $\operatorname{AUC}(0-\mathrm{inf})$

${ }^{1}$ The model-specific discrepancy in confidence for alectinib is not currently well understood

${ }^{2}$ Although ziprasidone qualifies as high confidence given AUC and $C_{\max }$ ratios of ratios which fall within bioequivalence criteria, the simulated, fed-state plasma concentration-time profile poorly captured observed data. As such, ziprasidone was qualified as moderate confidence

${ }^{3}$ Although clarithromycin model 2 demonstrated superior food effect prediction accuracy, model 2 required optimization to capture fasted clinical data. As model 1 utilized a purely bottom-up approach, confidence in that model is higher

${ }^{4}$ Simulation of clinical nefazodone concentration-time data initially resulted in overprediction, possibly explained by partial gastric emptying in vivo. Model 1 but not model 2 incorporated partial gastric emptying, explaining the final model-specific discrepancy in confidence

${ }^{5}$ The use of different methods to optimize individual segmental Peffs between models 1 and 2 may explain the model-specific discrepancy in confidence for trospium IR and XR formulations

2010) and converted into a human effective permeability, using the software's built-in conversion tool (Table I) (21). As nifedipine is metabolized mainly via CYP3A and undergoes pre-systemic metabolism, the extent of pre-systemic metabolism was fitted to match the observed AUC of nifedipine following oral administration of $5 \mathrm{mg}$ to fasted healthy subjects (41). Complete absorption was predicted for each dose level (5 mg, $10 \mathrm{mg}$, and $20 \mathrm{mg}$ ), which was in line with reported data $(42,43)$.

Following successful model verification, the pharmacokinetics of the $10 \mathrm{mg}$ nifedipine IR capsule were simulated under fed conditions. Predicted/observed AUC, $C_{\max }$, and 
Table III. Summary of the Proposed Mechanism of Food Effect and the Associated Confidence Category in the PBPK Prediction of Food Effect. Color Coding Indicates Confidence in the PBPK Food Effect Prediction; Green: High; Yellow: Moderate; Red: Low

\begin{tabular}{|c|c|c|c|c|}
\hline Compound & Food Effec & $\mathrm{t} B C S$ & Confidence in PBPK Prediction & Mechanism of Food Effect \\
\hline Alectinib & Positive & II & Low & Changes in microenvironment $\mathrm{pH}$ and complex effect of formulation \\
\hline Amiodarone & Positive & II & Low & Salt form \\
\hline Aprepitant & Positive & II/IV & High (middle-out) & Bile acids and phospholipids \\
\hline Cimetidine & None & III & High (middle-out) & No food effect \\
\hline Clarithromycin & None & II & Moderate & No food effect \\
\hline Dabrafenib & Negative & II & Low & Salt form; effect on microenvironment $\mathrm{pH}$ \\
\hline Danazol & Positive & II & Low & Uncertainty in solubility (in vivo) \\
\hline Danirixin & Negative & II & High (bottom-up) & Ion-pairing \\
\hline d-Sotalol & None & III & High (middle-out) & No food effect \\
\hline Etoricoxib & Negative & II & High (bottom-up) & GI motility changes in presence of food \\
\hline Fluoxetine $\mathrm{HCl}$ & None & I & High (bottom-up) & No food effect \\
\hline Furosemide & Negative & III & High (bottom-up) & GI motility changes in presence of food \\
\hline Imatinib & None & II & High (middle-out) & No food effect \\
\hline Isoniazid & Negative & I & Moderate & Drug-food interaction \\
\hline Itraconazole & Positive & II & High (middle-out) & Buffer capacity alters dissolution \\
\hline Ivacaftor & Positive & $\mathrm{II} / \mathrm{IV}$ & High (middle-out) & Bile acids and phospholipids \\
\hline Metoprolol & Positive & I & Moderate & Effect of hepatic and splanchnic blood flow \\
\hline Nefazodone $\mathrm{HCl}$ & Negative & II & Moderate & Effect of hepatic and splanchnic blood flow \\
\hline Nelfinavir Mesylate & Positive & $\mathrm{II} / \mathrm{IV}$ & Moderate & Precipitation kinetics affected by food \\
\hline Nifedipine & None & II & High (bottom-up) & No food effect \\
\hline Oseltamivir & None & III & Moderate & No food effect \\
\hline Panobinostat & None & II & High (bottom-up) & No food effect \\
\hline Pazopanib & Positive & II/IV & Low & Impact of biorelevant buffer species on solubilization*; Salt form \\
\hline Phenytoin & Positive & II & High (middle-out) & Bile acids and phospholipids \\
\hline Telaprevir & Positive & II & Low & Impact of biorelevant buffer species on solubilization ${ }^{*}$ \\
\hline Tezacaftor & None & II & High (middle-out) & No food effect \\
\hline Trospium IR/XR & Negative & III & Low & Changes in hydrodynamics (viscosity) in the presence of food \\
\hline Venetoclax & Positive & IV & Moderate & Lymphatic uptake \\
\hline Zidovudine & Negative & III & High (bottom-up) & GI motility changes in presence of food \\
\hline Ziprasidone $\mathrm{HCl}$ & Positive & II & Moderate & Salt form \\
\hline
\end{tabular}

*Specialized biorelevant media required to capture food effect

$T_{\max }$ ratios were $1.51,1.06$, and 1.00 , and thus within 2-fold of observed. As in the fasted state, absorption of nifedipine under fed conditions was predicted to be complete.

Additional examples of bottom-up with successful prediction of food effect risk without the need for optimization with clinical food effect data included etoricoxib (negative $\mathrm{FE}$ ), fluoxetine (no FE), and zidovudine (negative FE). Detailed descriptions of the modeling approach and outcome for these compounds are provided in an accompanying manuscript published in this issue.
PBPK Predictions to Waive Food Effect Studies-MiddleOut Application

\section{Case Example: Aprepitant (High Confidence)}

Aprepitant is a poorly soluble compound with moderate to high permeability and is non-ionized at intestinal $\mathrm{pH}$ values. Micronized aprepitant showed significant positive food effect (2.8-fold increase in AUC, 2.2-fold increase in $\left.C_{\max }\right)$ in healthy human volunteers given a high-fat breakfast. 


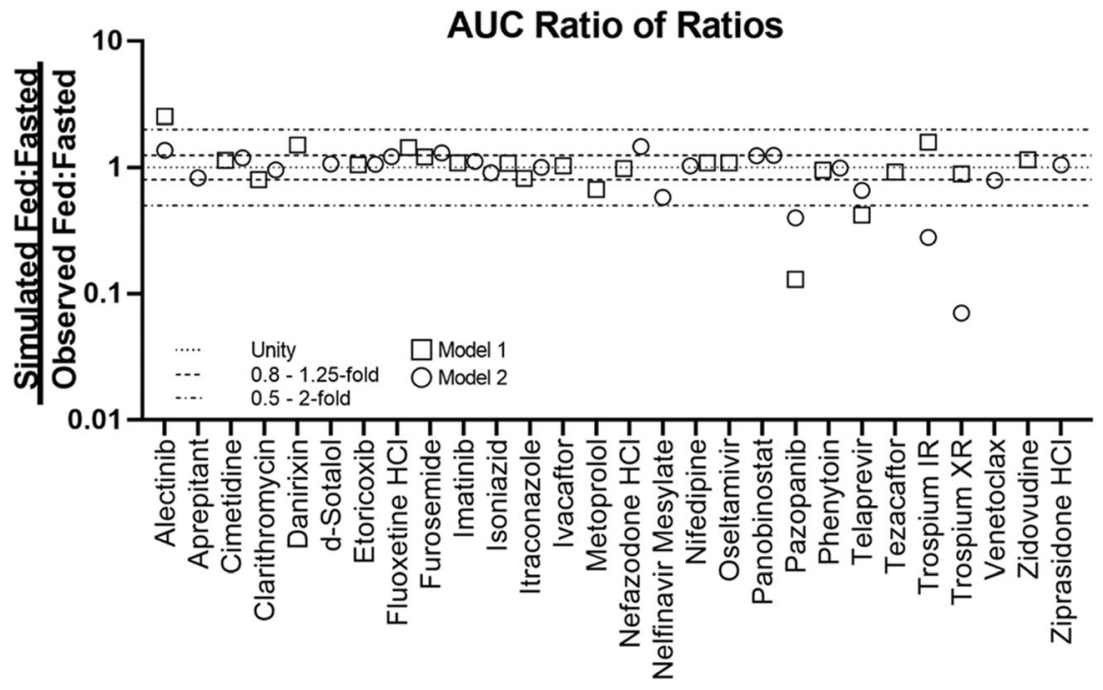

Fig. 3. AUC ratio of ratios for the modeled compounds. Models 1 and 2 refer to the two software programs used for prediction. Where confidence did not agree between the two software, the outcome from the model with lower confidence was used to assign confidence in the prediction

The bottom-up absorption model for the micronized formulation was built according to working group guidelines. Intrinsic and biorelevant solubility data of thermodynamically stable form and micronized drug substance were taken from literature as the drug substance was not commercially available and data was generated using the same approach as described above $(13,14)$. Partitioning of neutral and ionic species into bile micelles (i.e., $\log \mathrm{K}_{\mathrm{m}: \mathrm{w}}$ ) and DLM scalars were estimated using SIVA $(14,44,45)$. Clearance and disposition parameters were estimated from IV PK data (46).

The model was used to simulate oral PK of $100 \mathrm{mg}$ micronized aprepitant in fasted subjects. When the solubility measured in US Pharmacopeia Simulated Intestinal Fluids (SIFsp pH 6.8, $0.7 \mu \mathrm{g} / \mathrm{ml}$ ) was used as intrinsic solubility for simulations, PK parameters for the fasted condition were underpredicted (AUC predicted (3354 ng.hr./ml) vs observed
(8571 ng.h/ml), $C_{\max }$ predicted $(125 \mathrm{ng} / \mathrm{ml})$ vs observed (496 ng/ml). Therefore, an average of solubility values reported for thermodynamically stable form in water at $\mathrm{pH} 8.0(7 \mu \mathrm{g} / \mathrm{ml})$ and in $\mathrm{SIF}_{\mathrm{sp}}$ was used as intrinsic solubility which allowed for the prediction of $\mathrm{AUC}$ and $C_{\max }$ values within the pre-specified tolerance (i.e., AUC predicted $(11,142 \mathrm{ng} . \mathrm{h} / \mathrm{ml})$ vs observed $(8571 \mathrm{ng} . \mathrm{h} / \mathrm{ml}), C_{\max }$ predicted (455 $\mathrm{ng} / \mathrm{ml})$ vs observed $(496 \mathrm{ng} / \mathrm{ml}))$. The model was subsequently used to predict the effect of food on PK of a $100 \mathrm{mg}$ micronized aprepitant. The predicted increase in AUC and $C_{\max }$ was within $0.80-1.25$-fold of the observed values (AUC predicted $(26,673 \mathrm{ng} \cdot \mathrm{h} / \mathrm{ml})$ vs observed (24,057 ng.h/ml), $C_{\max }$ predicted $(1237 \mathrm{ng} / \mathrm{ml})$ vs observed (1098 ng/ml)) (44). Overall, the PBPK model was able to predict the observed positive food effect due to enhanced solubilization and dissolution.

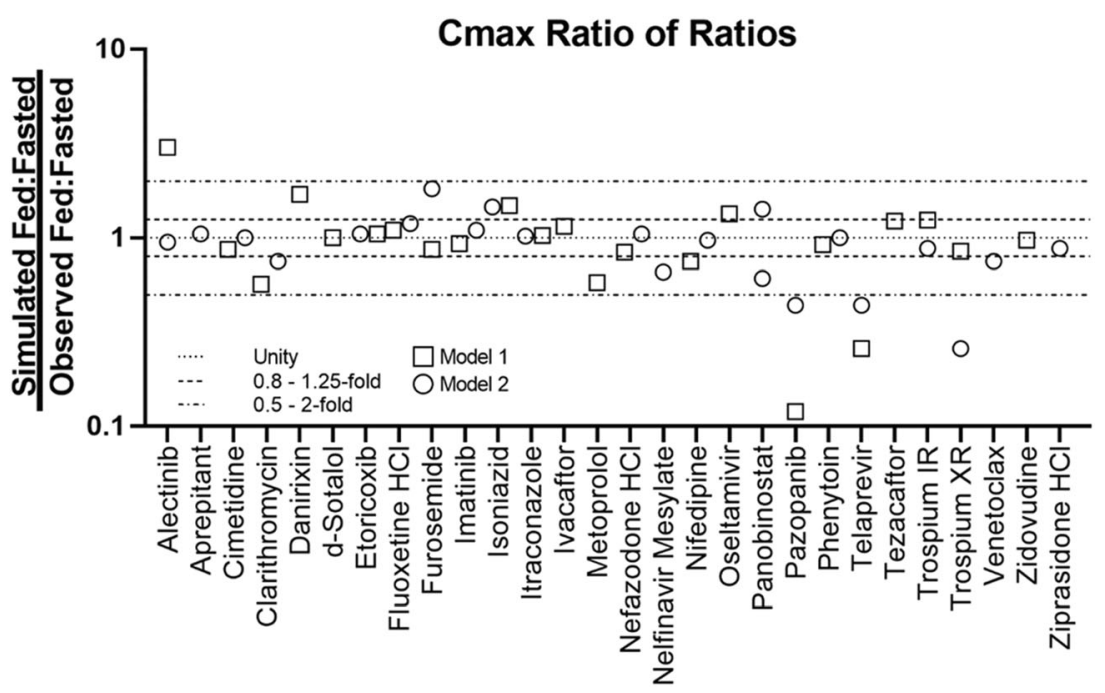

Fig. 4. $C_{\max }$ ratio of ratios for the modeled compounds. Models 1 and 2 refer to the two software programs used for prediction. Where confidence did not agree between the two software, the outcome from the model with lower confidence was used to assign confidence in the prediction 


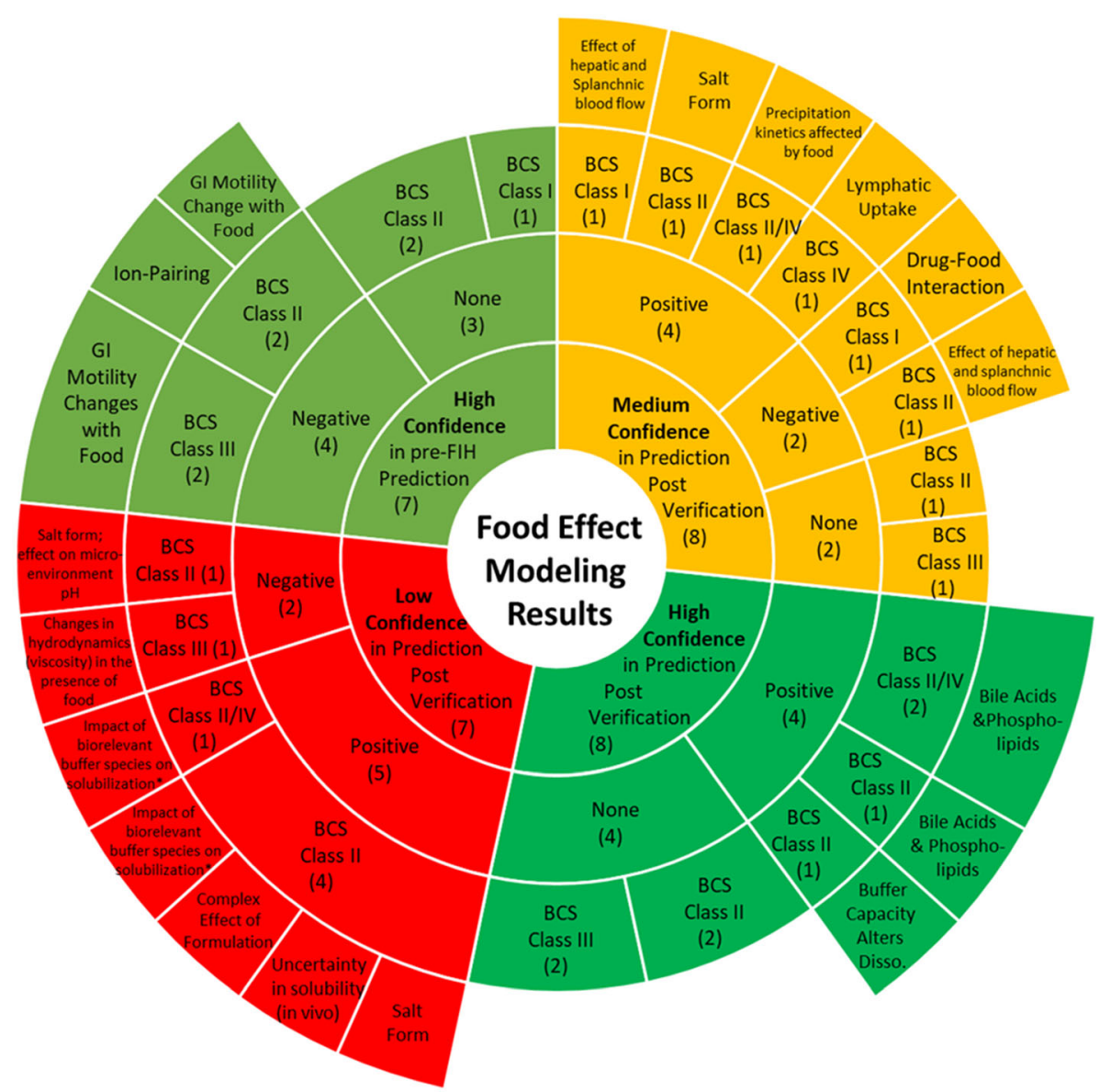

Fig. 5. Proposed mechanisms of food effect and their association with confidence in PBPK modeling for 30 modeled compounds. The inner layer of the plot depicts the confidence category, followed by direction of food effect in the second row, the BCS class in the third row, and the mechanism of food effect in the fourth row. The numbers in the first to third row indicate the number of compounds (out of 30) that fall in each category. More details around compound name and mechanism are provided in Table III

Additional examples with the successful prediction of food effect risk using a middle-out approach include furosemide (negative FE), nefazodone (negative FE), nelfinavir (positive FE), and phenytoin (positive FE). Detailed descriptions of the modeling approach and outcome for these compounds are provided in an accompanying manuscript published in this issue.

\section{Food Effect Predictions with Low Confidence}

\section{Case Example: Pazopanib}

Pazopanib is a BCS class II/IV compound (basic pKa 2.1 and 6.4) that exhibits low solubility across the physiological $\mathrm{pH}$ range. Clinically, pazopanib is dosed as the hydrochloride salt. At $800 \mathrm{mg}$ dose, fasted bioavailability was low and variable at $\sim 21 \%$ (range $14-39 \%$ ) and the compound exhibited a significant positive food effect (2.3-fold increase in $\mathrm{AUC}_{0-72 \mathrm{~h}}$ and 2.1-fold increase in $C_{\max }$ with a high-fat meal) (47). The compound is recommended to be taken without food (at least $1 \mathrm{~h}$ before or $2 \mathrm{~h}$ after a meal) (48).

Bottom-up absorption models were developed following the standardized workflow. Human permeability was projected based on Caco-2 data (Table I). Bile salt solubilization was estimated based on FaSSIF and FeSSIF data. The models (regardless of the software used) significantly underpredicted the fasted state plasma concentration profiles and did not predict the positive food effect. Both bottom-up models underpredicted the fasted state AUC at $800 \mathrm{mg}$ pazopanib-HCl by more than 2-fold. When the models were applied to predicting the effect of food on pazopanib's PK, they predicted a slight FE (AUC ratio fed/fasted $=0.86$ to 1.09), whereas the observed FE was approximately 2.3-fold. Further model optimization in accordance with the decision tree (Fig. 2) by adjusting the precipitation rate constant (essentially reducing in vivo precipitation) resulted in closer prediction of the fasted state profile (within 2-fold of AUC). However, the adjusted model failed to predict the significant positive food effect. Models predicted either no food effect or negative food effect, depending on the software used and the stomach $\mathrm{pH}$ settings. For both models, the default fed physiology was applied. Thus, the standardized optimization workflow failed to directionally replicate the observed food effect.

The inability to capture the in vivo pharmacokinetics of pazopanib may be due to challenges with modeling 
dissolution of salts that may be dissolving faster and/or to a greater extent than what the solubility measurements using standard methodologies suggest. Additionally, exploratory experiments suggest that the buffer species has a significant impact on the solubility and precipitation behavior of pazopanib (49). Dissolution measurements in FaSSIF and FeSSIF exhibited some supersaturation followed by precipitation. However, the translation of such in vitro observations to a PBPK setting is not straightforward. Additional discussion of pazopanib modeling as related to future opportunities in in vitro assays and model refinement are outlined below and a more detailed description of the modeling approach and outcome for pazopanib and trospium chloride is provided in an accompanying manuscript published in this issue.

\section{DISCUSSION}

In this study, a unique prospective approach was proposed to build and verify mechanistic PBPK models for 30 compounds while controlling for common variables, such as model input parameters, method of data generation, and subjective optimization and/or verification. To focus the model development and verification around absorption parameters, compounds with known IV clearance were used. However, we believe in practice the models can be applied even in the absence of IV data if the food effect mechanism is primarily related to absorption events. The focus of this work was to identify mechanisms where high, moderate, and low confidence in the prediction of food effect can be expected. The levels of confidence were defined based on modeling approach and an assessment of how well the PK parameters and profiles were captured. In total, the food effect of 15 compounds was predicted with high confidence, 8 with moderate confidence, and 7 with low confidence. Areas of high to moderate confidence were mainly associated with food effect related to changes in GI luminal fluid and physiology, while lower confidence was commonly associated with complex mechanisms and/or interplay between multiple mechanisms for which standardized in vitro assays and model input are not available to characterize the food effect, as well as to develop and verify models and/or gaps exist in capturing these mechanisms in the modeling software utilized.

Compound types where food effect was not appropriately captured using the standardized approach proposed in this work were salts and some weak bases, for which optimizing the precipitation time of the drug could not address the low confidence in capturing the absorption profile and extent of food effect. One key point for the misprediction of food effect is the current gaps in software and/or biorelevant media to adequately reflect the impact of food and food components for poorly soluble drugs; while biorelevant media are widely accepted for providing a good estimate of the luminal solubility of poorly soluble drugs under fasted and fed state, there seem to be cases where the in vitro solubility of these media is not in line with the solubility in human intestinal fluids (50), and cases where the buffer species has a pronounced impact on the in vitro solubility and precipitation behavior of certain compounds (49). Specifically, the outcome of our modeling exercise suggests that the in vivo solubility of pazopanib in the GI tract may be higher compared with the measured in vitro
FaSSIF solubility. Therefore, there is a need to further investigate more biorelevant media to improve PBPK-based absorption predictions at various prandial states.

In a typical clinical development paradigm, early assessment of food effect could be generated as early as the first-inhuman single ascending dose studies. In oncologic drug development, dosing with food (typically a light meal) may be pursued from the beginning, particularly if an increase in exposure is needed or GI-related toxicity can be mitigated. Under current global regulatory paradigms, most companies are expected to re-characterize the food effect on the to-bemarketed formulation. Tistaert et al. previously proposed that following validation of the model against fasted and fed data that have been generated in early development, one could apply the model prospectively to account for food effect of new doses, formulations, or API forms (9). In this manuscript, we have built on these recommendations by highlighting opportunities where we find the translation of these models across formulations may be appropriate and where in vitro methodology and PBPK models may require further advancements in their approach to be adequately predictive. When the mechanisms of food effect can be categorized as high confidence, bottom-up, the PBPK models may be used for decision-making around food effect prior to FIH and also, it may not be required to perform a clinical anchor study (i.e., a specific food effect study in healthy volunteers under wellcontrolled conditions and powered to show bioequivalence). In such cases, the high confidence PBPK FE projections could still be confirmed with clinical data (e.g., an arm of fed subjects in a Ph1 study or a PopPK analysis of patient data) (51). It is also worth noting that the relevant food effect in clinical use in the patient population may differ from that measured in an anchor study in healthy volunteers with a standard meal. Such drugs may include compounds where solubility is generally not rate-limiting and food effect (typically negative for $C_{\max }$ ) is primarily dictated by gastric emptying.

Mechanisms categorized as high confidence, middle-out may need to be verified using an anchor study before being used to predict food effect with high accuracy. However, there is still the possibility to avoid additional clinical studies around food effect after certain formulation or dose changes. Similarly, when models and mechanisms categorized as having moderate confidence (within 2-fold) are verified with an anchor study, there may be cases where minor formulation changes can be made without the need for additional FE studies. At this point, the nature of the formulation change should be considered. Formulation changes are commonplace during clinical development. While, on some occasions, major formulation changes may be pursued to address specific clinical needs (e.g., an "enabled" formulation to address the poor bioavailability of an early crystalline formulation), most formulation changes undertaken are more subtle. The formulation technology is typically decided early on and optimization focuses on the composition and manufacturing process. Based on the work presented in this manuscript, we propose that if the formulations follow the same dissolution/ absorption principles (e.g., both formulations are based on crystalline API), translation of the models from early to late stage is possible especially when the mechanism of food effect (or lack thereof) is well understood (i.e., the defined 
confidence categories should hold). For many of the examples highlighted in this work, the formulation dissolution rate is not the determining factor for food effect. For rapiddissolving formulations, food effect may be dictated by gastric emptying time (if sufficient solubility or administered as nonprecipitating oral solution) or by the saturation solubility of the API in the fasted and fed condition. This is mostly independent of the formulation and would not be affected by many formulation changes. However, the authors acknowledge that the proposal to avoid clinical studies for minor formulation changes has been supported by only limited published examples $(9,52)$ and further work is recommended to verify this with a broader range of clinical data. While the primary application of such models is to describe the food effect in the context of biopharmaceutics characterization, on a case-by-case basis, these models can be considered for prospective application dependent on therapeutic margins and exact mechanism of food effect verified with the anchor study.

Like formulation changes, we believe that PBPK models could be applied to the projection of food effect at different doses than previously established. A properly qualified PPBK model across different doses would accurately account for absorption limitations (if applicable) and thus would be wellpositioned to assess the impact of food at different doses than previously studied. An interesting question would be the application of food effect models to different meals (e.g., a moderate-fat, moderate-calorie meal). There are currently a relatively limited number of published examples systematically looking at parameterization of the models as a function of the meal content. The most detailed example is probably the study by Sutton et al. (53) where the authors proposed adjustments to the gastric emptying and bile salt concentration settings in the PBPK model for different meals. Adopting such a model may be considered to interpolate exposures between the typically tested prandial states (i.e., fasted and high-fat/high-calorie meal). While this could help with simulating more real-life dosing conditions, it was out of scope of the work presented here.

Food effect prediction with a PBPK model seamlessly integrates in vitro data with knowledge of human physiology. Prior work has attempted food effect prediction from only in vitro data (54-56) or directly from animal models (56-58). While these methods have shown some success in predicting the direction of food effect, their ability to accurately capture the extent of food effect, and the impact of food on PK parameters and, especially, profiles have not been consistently demonstrated. While it makes sense to use all available data, including pre-clinical data (e.g., dog FE data), to inform predictions of human food effect, the focus of this study was to explore the predictive ability of PBPK models following a pure in vitro to in vivo approach. We present the application of standard PBPK platforms to accurately capture not only the direction of food effect but also the extent of the effect on the human PK profile. Given the complex nature of food effect, it is anticipated that an integrated approach such as PBPK, which captures the complexity of human physiology and disparate food effect mechanism, can serve as a key platform for the support of food effect predictions.

The study of food effect on the absorption of orally administered drugs is widespread and a key driver of study design, data analysis, and labeling language. Within the pharmaceutical industry, significant resources are invested to predict and characterize a clinically meaningful food effect, including the use of PBPK models to gain mechanistic insight into potential food effect. Here, the predictive performance of PBPK food effect models was assessed using de novo mechanistic absorption models for 30 compounds generated in accordance with controlled, pre-defined in vitro and modeling methodology, as well as an aligned decision tree for model design, verification, and optimization. Mechanistic PBPK models enabled food effect modeling of most of the compounds with high $(50 \%)$ or moderate $(27 \%)$ confidence, with a small subset of compounds showing low (23\%) confidence in the prediction of food effect. A correlation was observed between the confidence in the model and the mechanism of food effect, whereby models generally showed high confidence in prediction where food effect was related to changes in GI fluid volume, motility, or luminal fluid composition, while food effects related to drug interactions with intestinal microenvironment and/or food-drug/micelledrug interactions were more difficult to predict with high confidence. This analysis did not include prodrugs and compounds whose absorption is known to be limited by intestinal active transport; however, such compounds are not expected to make up a significant subset of clinical compounds displaying food effect. While the correlation between model confidence and mechanism of food effect was established for 30 compounds in this study, it is only the first step in understanding this correlation. Future work should focus on further strengthening the validity of these conclusions by expanding this analysis to additional compounds.

\section{CONCLUSION}

Considering these findings, it is recommended that appropriately verified, mechanistic PBPK models be leveraged as a key approach to studying potential food effect, especially related to mechanisms associated with high to moderate confidence, thereby replacing the need to conduct clinical food effect studies.

\section{ACKNOWLEDGMENTS}

The authors would like to thank David Lindley for his initiative, valuable guidance, and contribution to the solubility and dissolution data generation, which were instrumental to the PBPK modeling. Additional thanks to Zhizhou Fang for his contribution to the MDCK permeability data generation.

Open Access This article is licensed under a Creative Commons Attribution 4.0 International License, which permits use, sharing, adaptation, distribution and reproduction in any medium or format, as long as you give appropriate credit to the original author(s) and the source, provide a link to the Creative Commons licence, and indicate if changes were made. The images or other third party material in this article are included in the article's Creative Commons licence, unless indicated otherwise in a credit line to the material. If material is not included in the article's Creative Commons licence and your intended use is not permitted by statutory regulation or 
exceeds the permitted use, you will need to obtain permission directly from the copyright holder. To view a copy of this licence, visit http://creativecommons.org/licenses/by/4.0/.

\section{REFERENCES}

1. Fleisher D, Li C, Zhou Y, Pao LH, Karim A. Drug, meal and formulation interactions influencing drug absorption after oral administration. Clin Pharmacokinet. 1999;36(3):233-54.

2. O'Shea JP, Holm R, O'Driscoll CM, Griffin BT. Food for thought: formulating away the food effect - a PEARRL review. J Pharm Pharmacol. 2019;71(4):510-35.

3. FDA. Assessing the Effects of Food on Drugs in INDs and NDAs - Clinical Pharmacology Considerations Guidance for Industry. 2019; Available from: https://www.fda.gov/media/ 121313/download.

4. EMA. Guideline on the Investigation of Drug Interactions. 2012.

5. Koziolek M, Alcaro S, Augustijns P, Basit AW, Grimm M, Hens $\mathrm{B}$, et al. The mechanisms of pharmacokinetic food-drug interactions - a perspective from the UNGAP group. Eur J Pharm Sci. 2019;134:31-59.

6. Pentafragka C, Symillides M, McAllister M, Dressman J, Vertzoni M, Reppas C. The impact of food intake on the luminal environment and performance of oral drug products with a view to in vitro and in silico simulations: a PEARRL review. J Pharm Pharmacol. 2019;71(4):557-80.

7. Emami Riedmaier A, Lindley DJ, Hall JA, Castleberry S, Slade RT, Stuart P, et al. Mechanistic physiologically based pharmacokinetic modeling of the dissolution and food effect of a biopharmaceutics classification system IV compound-the Venetoclax story. J Pharm Sci. 2018;107(1):495-502.

8. Li M, Zhao P, Pan Y, Wagner C. Predictive performance of physiologically based pharmacokinetic models for the effect of food on oral drug absorption: current status. CPT Pharmacometrics Syst Pharmacol. 2018;7(2):82-9.

9. Tistaert C, Heimbach T, Xia B, Parrott N, Samant TS, Kesisoglou F. Food effect projections via physiologically based pharmacokinetic modeling: predictive case studies. J Pharm Sci. 2019:108(1):592-602.

10. Wagner C, Zhao P, Pan Y, Hsu V, Grillo J, Huang SM, et al. Application of physiologically based pharmacokinetic (PBPK) modeling to support dose selection: report of an FDA public workshop on PBPK. CPT Pharmacometrics Syst Pharmacol. 2015;4(4):226-30.

11. Parrott NJ, Yu LJ, Takano R, Nakamura M, Morcos PN. Physiologically based absorption modeling to explore the impact of food and gastric $\mathrm{pH}$ changes on the pharmacokinetics of alectinib. AAPS J. 2016;18(6):1464-74.

12. Meng X, Mojaverian P, Doedée M, Lin E, Weinryb I, Chiang ST, et al. Bioavailability of amiodarone tablets administered with and without food in healthy subjects. Am J Cardiol. 2001;87(4):432-5.

13. Wu Y, Loper A, Landis E, Hettrick L, Novak L, Lynn K, et al. The role of biopharmaceutics in the development of a clinical nanoparticle formulation of MK-0869: a beagle dog model predicts improved bioavailability and diminished food effect on absorption in human. Int J Pharm. 2004;285(1-2):135-46.

14. Shono Y, Jantratid E, Kesisoglou F, Reppas C, Dressman JB. Forecasting in vivo oral absorption and food effect of micronized and nanosized aprepitant formulations in humans. Eur $\mathbf{J}$ Pharm Biopharm. 2010;76(1):95-104.

15. FDA. Clinical Pharmacology NDA Review for Tafinlar (Dabrafenib). NDA 202806/3. 2013

16. Childs SL, Kandi P, Lingireddy SR. Formulation of a danazol cocrystal with controlled supersaturation plays an essential role in improving bioavailability. Mol Pharm. 2013;10(8):3112-27.

17. Guo J, Elzinga PA, Hageman MJ, Herron JN. Rapid throughput solubility screening method for BCS class II drugs in animal GI fluids and simulated human GI fluids using a 96-well format. J Pharm Sci. 2008;97(4):1427-42.
18. Sunesen VH, Pedersen BL, Kristensen HG, Müllertz A. In vivo in vitro correlations for a poorly soluble drug, danazol, using the flow-through dissolution method with biorelevant dissolution media. Eur J Pharm Sci. 2005;24(4):305-13.

19. Villiger A, Stillhart C, Parrott N, Kuentz M. Using physiologically based pharmacokinetic (PBPK) modelling to gain insights into the effect of physiological factors on oral absorption in paediatric populations. AAPS J. 2016;18(4):933-47.

20. Mitra A, Kesisoglou F, Dogterom P. Application of absorption modeling to predict bioequivalence outcome of two batches of etoricoxib tablets. AAPS PharmSciTech. 2015;16(1):76-84.

21. Lennernas H. Regional intestinal drug permeation: biopharmaceutics and drug development. Eur J Pharm Sci. 2014;57:333-41.

22. Takacs-Novak K, et al. Biorelevant solubility of poorly soluble drugs: rivaroxaban, furosemide, papaverine and niflumic acid. J Pharm Biomed Anal. 2013;83:279-85.

23. Flanagan SD, Takahashi LH, Liu X, Benet LZ. Contributions of saturable active secretion, passive transcellular, and paracellular diffusion to the overall transport of furosemide across adenocarcinoma (Caco-2) cells. J Pharm Sci. 2002;91(4):1169-77.

24. Wiest J, Saedtler M, Böttcher B, Grüne M, Reggane M, Galli B, et al. Geometrical and structural dynamics of imatinib within biorelevant colloids. Mol Pharm. 2018;15(10):4470-80.

25. Andreas CJ, Pepin X, Markopoulos C, Vertzoni M, Reppas C, Dressman JB. Mechanistic investigation of the negative food effect of modified release zolpidem. Eur J Pharm Sci. 2017;102:284-98.

26. Lukacova V, Woltosz WS, Bolger MB. Prediction of modified release pharmacokinetics and pharmacodynamics from in vitro, immediate release, and intravenous data. AAPS J. 2009;11(2):323-34.

27. Longer M, Zamansky I, Tyle P, Shetty B. Preformulation studies of a novel HIV protease inhibitor, AG1343. J Pharm Sci. 1995;84(9):1090-3.

28. Shono Y, Jantratid E, Dressman JB. Precipitation in the small intestine may play a more important role in the in vivo performance of poorly soluble weak bases in the fasted state: case example nelfinavir. Eur J Pharm Biopharm. 2011;79(2):349-56.

29. Wagner C, Thelen K, Willmann S, Selen A, Dressman JB. Utilizing in vitro and PBPK tools to link ADME characteristics to plasma profiles: case example nifedipine immediate release formulation. J Pharm Sci. 2013;102(9):3205-19.

30. Gertz M, Harrison A, Houston JB, Galetin A. Prediction of human intestinal first-pass metabolism of 25 CYP3A substrates from in vitro clearance and permeability data. Drug Metab Dispos. 2010;38(7):1147-58.

31. Parrott N, Davies B, Hoffmann G, Koerner A, Lave T, Prinssen E, et al. Development of a physiologically based model for oseltamivir and simulation of pharmacokinetics in neonates and infants. Clin Pharmacokinet. 2011;50(9):613-23.

32. FDA. Clinical Pharmacology Review NDA $22-465$ for Votrient (Pazopanib). 2008.

33. Pade V, Stavchansky S. Link between drug absorption solubility and permeability measurements in Caco-2 cells. J Pharm Sci. 1998;87(12):1604-7.

34. Tadken T, Weiss M, Modess C, Wegner D, Roustom T, Neumeister C, et al. Trospium chloride is absorbed from two intestinal "absorption windows" with different permeability in healthy subjects. Int J Pharm. 2016;515(1-2):367-73.

35. Radwan A, Amidon GL, Langguth P. Mechanistic investigation of food effect on disintegration and dissolution of BCS class III compound solid formulations: the importance of viscosity. Biopharm Drug Dispos. 2012;33(7):403-16.

36. Yee S. In vitro permeability across Caco-2 cells (colonic) can predict in vivo (small intestinal) absorption in man-fact or myth. Pharm Res. 1997;14(6):763-6.

37. Vertzoni M, et al. Simulation of fasting gastric conditions and its importance for the in vivo dissolution of lipophilic compounds. Eur J Pharm Biopharm. 2005;60:413-7.

38. Jantratid E, Janssen N, Reppas C, Dressman JB. Dissolution media simulating conditions in the proximal human gastrointestinal tract: an update. Pharm Res. 2008;25(7):1663-76. 
39. Jantratid E, Dressman J. Biorelevant dissolution media simulating the proximal human gastrointestinal tract: an update. Dissolut Technol. 2009;16(3):21-5.

40. Reitberg DP, Love SJ, Quercia GT, Zinny MA. Effect of food on nifedipine pharmacokinetics. Clin Pharmacol Ther. 1987;42(1):72-5.

41. Raemsch KD, Sommer J. Pharmacokinetics and metabolism of nifedipine. Hypertension. 1983;5(1):18-24.

42. Chung M, Reitberg DP, Gaffney M, Singleton W. Clinical pharmacokinetics of nifedipine gastrointestinal therapeutic system: a controlled-release formulation of nifedipine. Am J Med. 1987;83(6):10-4.

43. Bode H, Brendel E, Ahr G, Fuhr U, Harder S, Staib AH. Investigation of nifedipine absorption in different regions of the human gastrointestinal (GI) tract after simultaneous administration of 13C- and 12C-nifedipine. Eur J Clin Pharmacol. 1996;50(3):195-201.

44. Georgaka D, Butler J, Kesisoglou F, Reppas C, Vertzoni M. Evaluation of dissolution in the lower intestine and its impact on the absorption process of high dose low solubility drugs. Mol Pharm. 2017;14(12):4181-91.

45. Pathak SM, Ruff A, Kostewicz ES, Patel N, Turner DB, Jamei M. Model-based analysis of biopharmaceutic experiments to improve mechanistic oral absorption modeling: an integrated in vitro in vivo extrapolation perspective using ketoconazole as a model drug. Mol Pharm. 2017;14(12):4305-20.

46. Majumdar AK, Howard L, Goldberg MR, Hickey L, Constanzer M, Rothenberg PL, et al. Pharmacokinetics of aprepitant after single and multiple oral doses in healthy volunteers. J Clin Pharmacol. 2006;46(3):291-300.

47. Verheijen RB, Beijnen JH, Schellens JHM, Huitema ADR, Steeghs N. Clinical pharmacokinetics and pharmacodynamics of pazopanib: towards optimized dosing. Clin Pharmacokinet. 2017;56(9):987-97.

48. VOTRIENT highlights of prescribing information, accessed online at on 26-Jul-2019. [cited 2019 26-Jul]. Available from: h t t p s :// w w w. pharma.us.novartis.com/sites/ www.pharma.us.novartis.com/files/votrient.pdf.

49. Jede C, Wagner C, Kubas H, Weigandt M, Weber C, Lecomte $\mathrm{M}$, et al. Improved prediction of in vivo supersaturation and precipitation of poorly soluble weakly basic drugs using a biorelevant bicarbonate buffer in a gastrointestinal transfer model. Mol Pharm. 2019;16(9):3938-47.

50. Söderlind E, et al. Simulating fasted human intestinal fluids: understanding the roles of lecithin and bile acids. Mol Pharm. 2010;7(5):1498-507.

51. Lu T, Fraczkiewicz G, Salphati L, Budha N, Dalziel G, Smelick GS, et al. Combining "bottom-up" and "top-down" approaches to assess the impact of food and gastric $\mathrm{pH}$ on pictilisib (GDC0941) pharmacokinetics. CPT Pharmacometrics Syst Pharmacol. 2017;6(11):747-55.

52. Parrott N, Hainzl D, Scheubel E, Krimmer S, Boetsch C, Guerini E, et al. Physiologically based absorption modelling to predict the impact of drug properties on pharmacokinetics of bitopertin. AAPS J. 2014;16(5):1077-84.

53. Sutton SC, Nause R, Gandelman K. The impact of gastric pH, volume, and emptying on the food effect of ziprasidone oral absorption. AAPS J. 2017;19(4):1084-90.

54. $\mathrm{Gu} \mathrm{C}$, et al. Predicting effect of food on extent of drug absorption based on physicochemical properties. Pharm Res. 2007;24(6):1118-30.

55. Sugano K, Kataoka M, da Costa Mathews C, Yamashita S. Prediction of food effect by bile micelles on oral drug absorption considering free fraction in intestinal fluid. Eur J Pharm Sci. 2010;40(2):118-24.

56. Mathias N, Xu Y, Vig B, Kestur U, Saari A, Crison J, et al. Food effect in humans: predicting the risk through in vitro dissolution and in vivo pharmacokinetic models. AAPS J. 2015;17(4):98898.

57. Christiansen ML, et al. Evaluation of the use of göttingen minipigs to predict food effects on the oral absorption of drugs in humans. J Pharm Sci. 2014.

58. Kondo H, Shinoda T, Nakashima H, Watanabe T, Yokohama S. Characteristics of the gastric $\mathrm{pH}$ profiles of unfed and fed cynomolgus monkeys as pharmaceutical product development subjects. Biopharm Drug Dispos. 2003;24(1):45-51.

Publisher's Note Springer Nature remains neutral with regard to jurisdictional claims in published maps and institutional affiliations. 